\title{
Measuring topographies from conventional SEM acquisitions
}

\author{
Qiwei Shi ${ }^{\mathrm{a}, \mathrm{b}}$, Stéphane Roux ${ }^{\mathrm{a}}$, Félix Latourte $^{\mathrm{b}}$, François Hild $^{\mathrm{a}}$, Dominique Loisnard ${ }^{\mathrm{b}}$, Nicolas Brynaert $^{\mathrm{b}}$ \\ ${ }^{a} L M T$, ENS Paris-Saclay / CNRS / Université Paris-Saclay \\ 61 avenue du Président Wilson, 94235 Cachan (FRANCE) \\ ${ }^{b} E D F$ R\&D, Site des Renardières, \\ avenue des Renardières, Ecuelles, 77818 Moret-sur-Loing (FRANCE)
}

\begin{abstract}
The present study extends the stereoscopic imaging principle for estimating the surface topography to two orientations, namely, normal to the electron beam axis and inclined at $70^{\circ}$ as suited for EBSD analyses. In spite of the large angle difference, it is shown that the topography can be accurately determined using regularized global Digital Image Correlation. The surface topography is compared to another estimate issued from a 3D FIB-SEM procedure where the sample surface is first covered by a Pt layer, and its initial topography is progressively revealed from successive FIB-milling. These two methods are successfully compared on a $6 \%$ strained steel specimen in an in situ mechanical test. This analysis is supplemented by a third approach estimating the change of topography from crystal rotations as measured from successive EBSD images. This last technique ignores plastic deformation, and thus only holds in an elastic regime. For the studied example, despite the large plastic flow, it is shown that crystal rotation already accounts for a significant part of the deformation-induced topography.
\end{abstract}

Keywords: Crystal plasticity, Digital image correlation, Focused Ion Beam, SEM, Topography

\section{Introduction}

Surface topography is critical for many properties, especially for mechanically demanding applications. For example, fatigue failure and corrosion resistance are often controlled by surface roughness, and therefore the latter is carefully controlled in many products from dental implants [1] to workpiece materials [2]. Topography measurements are also crucial for inspecting and analyzing defects in semiconductor products [3]. They also help to study the plastic properties of materials. For example, topography changes due to crystallographic slip have been assessed quantitatively by AFM and qualitatively by SEM to characterize plastic deformations at very local levels [4, 5]. The topography measurement of nanoindentation with AFM helps for the direct observation of plasticity for different crystal planes [6]. Recent studies show that the precise measurement of the 3D material microstructure and its subsequent deformation, thus including topography, improves identification results of crystal plasticity parameters [7, 8].

To characterize the surface topography and/or its changes during a mechanical test in the nano- to micrometer range, it is quite demanding to perform both mechanical test in situ and at the same time utilize different techniques to measure topography. Thus using a single instrument appears quite attractive. SEMs, through their variety of modalities, offer several ways to evaluate the surface geometry. A taxonomy of 3D SEM surface reconstructions can be found in Ref. [9]. Not all techniques offer the same reliability and measurement quality:

- FIB offers remarkable opportunities to perform nano-machining [10] or material deposition [11]. The successive removal of surface layers by FIB followed by EBSD analyses allows a 3D volume with crystallographic information to be reconstructed (i.e., a technique called 3D-EBSD [12]), which opened new 
avenues for characterizing materials in three-dimensions [13, 14, 15] (alas destructively). In particular, the surface topography can be measured through the very accurate estimate of successive contour lines.

- In the 1980s, photogrammetry, also known as stereomatching, provided quantitative results thanks to the advent of Digital Image Correlation (DIC) [16. From a pair of images of a specimen acquired at different inclination angles, the sample elevation was measured from the registration of the two images by DIC. During the last decades both DIC and pattern marking for SEM images have known significant developments. Thus measurement reliability and accuracy have improved and their application spectrum has been broadened. The uncertainty of the stereoscopic analysis of SEM images has been quantified [17]. Various types of errors in the measurement process have been studied and partly corrected [18, including SEM distortion, system calibration and tilt angle inaccuracy. For surfaces between the identified matching points, an interpolation is necessary to obtain an estimation of topography. Delaunay triangulation and linear interpolation have been adopted [19, thereby resulting in a surface made of small facets of different normal directions [20]. Thus the density of matching points dictates the surface representation. One major challenge is the accurate matching of all points between images since a single error will bias the obtained topography [16]. Neural adaptive learning or machine learning have been successfully applied to ensure matching [21, 22. Yet such approaches remain fragile as long as no assessment of the registration quality is available.

- Shape-from-shading is another method that was proposed in the 1970s [23, 24] and revisited many times. This approach exploits the dependence of the secondary or backscattered electron intensity on the surface orientation and detector position [25]. The use of multi-detectors has been proposed to perform topographic reconstruction in an SEM without a physical rotation of the sample. Thus only gray level differences are exploited, and not detailed registration. The difficulty is the quantitative interpretation of these gray level variations, in particular for the SE mode, in spite of their better spatial resolution [26]. At present, this technique is mostly qualitative.

The more recent SEMs can operate on tilted samples thanks to their enhanced depth of focus and better control of e-beam collimation. This is in particular exploited in EBSD analyses where the sample is generally tilted to about $70^{\circ}$. In the present study, the stereoscopic approach is followed to evaluate the surface topography from two orientations. The latter are chosen as the ones corresponding to these standard acquisition modes (i.e., normal to the beam axis and in the EBSD-tilt orientation). Although the principle of stereoscopic topography reconstruction is classical, such orientations are very unusual. To tackle this problem, regularized global DIC is used to register images after having revealed a speckle pattern consisting of Pt dots deposited onto the surface. This procedure allows the changes in gray levels due to the very large tilt angle to have no influence on the quality of registration. The sample used as a support for this study is a steel specimen with a polished surface, but subjected in situ to a mechanical test up to $6 \%$ macroscopic strain. This method and result of such topography measurements are detailed in Section 2 .

In order to assess the quality of the surface topography measurement, 3D-EBSD is used, where only surface topography is considered herein for comparison purposes. The procedure and results are detailed in Section 3 . A third original approach is presented that aims at capturing the change in topography as resulting from crystal rotations only (Section 4.1]. Crystal rotation fields are measured from a series of EBSD images acquired at different stages of deformation via so-called "quaternion correlation," which was proposed to capture the grain boundary kinematics in polycrystalline solids [27]. Based on the sole crystallography, grain rotations can explain part of the out-of-plane surface displacements, and hence topography formation. However, plastic flow does 
not alter the crystal orientation and hence cannot be assessed with this tool. Conversely, it allows the relative part of crystal rotation vs. plastic flow in the formation of topography to be estimated. In the present case, it is shown that in spite of significant plastic flow, crystal rotations (as measured herein) capture already a significant part of the topography. The relative merits and weaknesses of all these approaches are discussed in Section 5 .

\section{SEM tilt-imaging method}

\subsection{Mechanisms}

The principle of stereoscopic topography evaluation is well-known [28]. From images of the surface under two orientations, the topography can be reconstructed. Generally the two orientations are chosen as tilted from the electron beam axis by $\pm 10^{\circ}[29,17,18$. Here, this technique is applied to two orientations, namely, in the first one the mean surface normal is parallel to the e-beam axis, while the second is tilted to roughly $70^{\circ}$ in the standard EBSD configuration (Figure 1). This choice is made because these two orientations are often accessible when an EBSD analyzer is present. However, such a very large tilt angle is not used for stereoscopic analyses, as large distortions and shadowing effects may be feared. Moreover, the fragility of missing matching points as earlier mentioned is also expected to be increased in such a configuration. It will be shown that regularized global DIC restores the needed robustness for such a technique to be safely used.

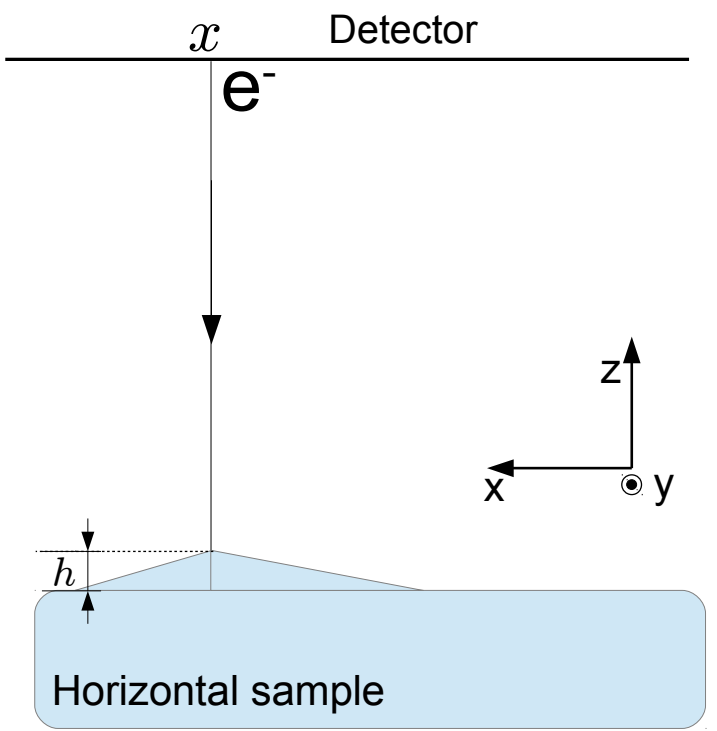

(a)

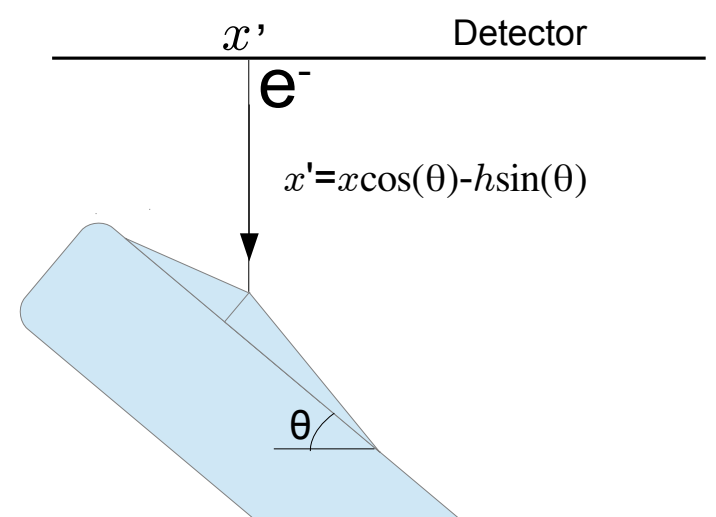

Tilted sample

Figure 1: Mechanism of the tilt-imaging method. SEM image of the sample on horizontal (a) and tilted (b) positions by angle $\theta$.

Since the adopted SEM magnification is high (i.e., $\times 6400$ ), the e-beam is considered as parallel [16]. In the horizontal configuration, the sample surface is denoted as $z=h(x, y)$. The tilted configuration is obtained after a rotation of angle $\theta$ about the $y$ axis, as shown in Figure 1 .

In the tilted configuration, the point $(x, y, z)$ is mapped horizontally at a position $\left(x^{\prime}, y^{\prime}, z^{\prime}\right)$, where

$$
x^{\prime}=x \cos (\theta)-h(x, y) \sin (\theta)
$$

and $y^{\prime}=y$. Typically, for a small roughness, $h \ll x$, the main effect of tilt is a mere affinity, by a factor $\cos (\theta)$ (i.e., just as if $h=0$ in the above equation). Compensating for this scale factor by an inverse affine transformation, $x^{\prime \prime}=x^{\prime} / \cos (\theta)$, moves the point to a position $x^{\prime \prime}=x-h(x, y) \tan (\theta)$. Hence the topography simply translates into a displacement $u_{x}=h(x, y) \tan (\theta)$. 


\subsection{Uncertainty quantification}

Before applying the tilt-imaging method to the real topography, an uncertainty evaluation has been performed. Pt speckles have been deposited at prescribed positions on a Ge single crystal sample with a polished surface. Each Pt speckle is a disk whose diameter is $300 \mathrm{~nm}$ and height $75 \mathrm{~nm}$. The sample surface is assumed to be perfectly flat and the measured topography is attributed to errors. The first image has been taken with the Ge sample in the horizontal position (see Figure 2(a) , and the second image when the sample has been inclined at $70^{\circ}$ (see Figure 2(b) . The image definition is $2048 \times 2048$ pixels, and covers an area of $110 \times 110 \mu \mathrm{m}^{2}$. The images have been acquired with an acceleration voltage of $30 \mathrm{kV}$ and a working distance of $16 \mathrm{~mm}$. To reduce the possible SEM drift distortion, each SEM image is the average result of 30 scanned frames. The scan speed is set to 3 , which corresponds to a dwell time of 1 s to lower the image acquisition duration. With these settings, it has taken 2 minutes to get an image. Global DIC with mechanical regularization has been applied on the image pair, and the resulting displacement fields in $x$ and $y$ directions are shown respectively in Figures 2(c) and $2(\mathrm{~d})$. 


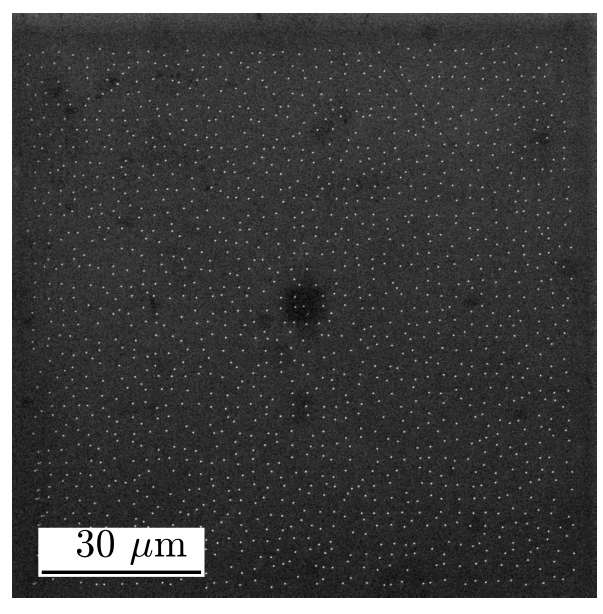

(a)

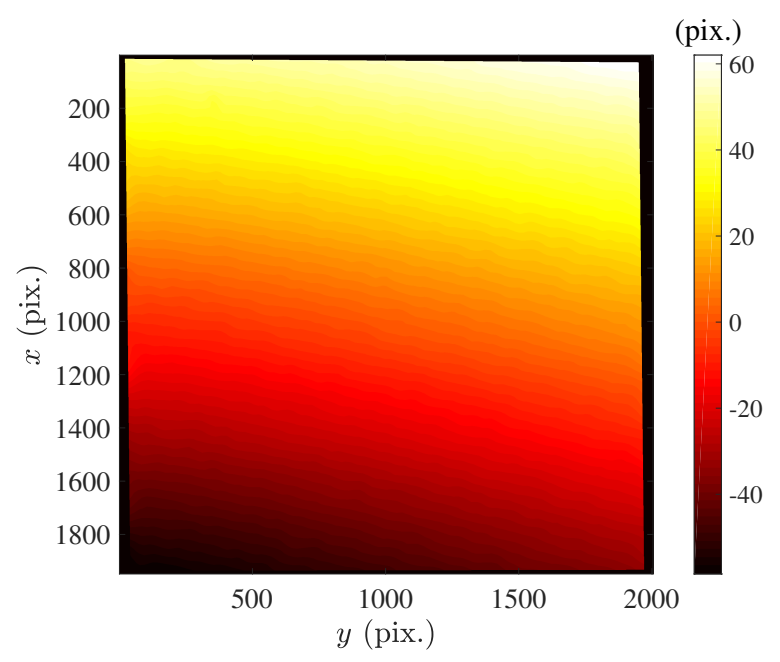

(c)

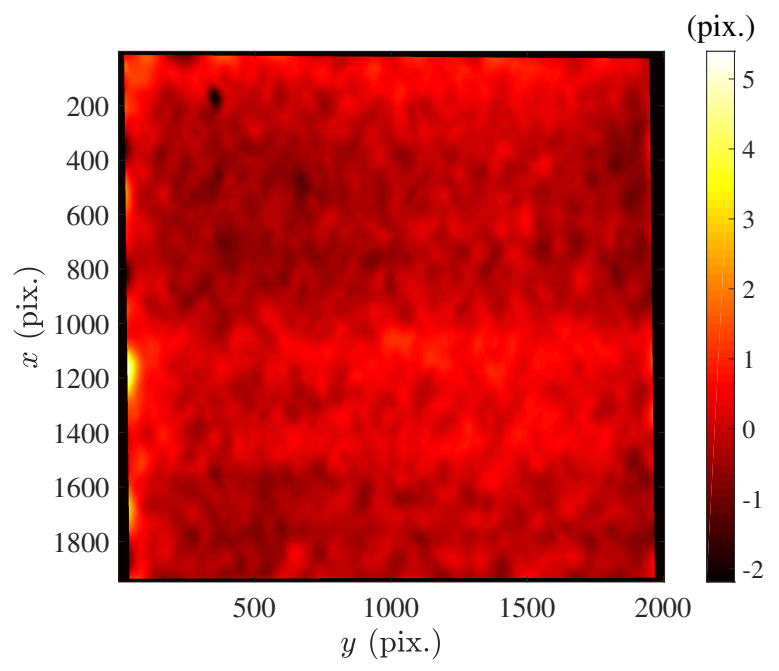

(e)

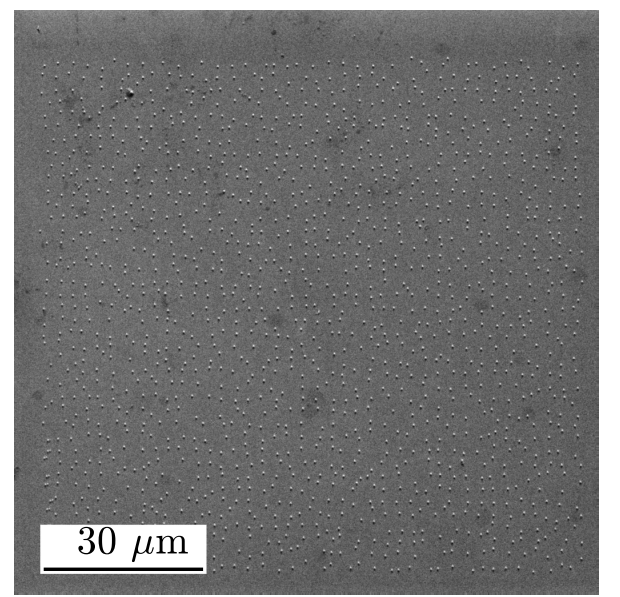

(b)

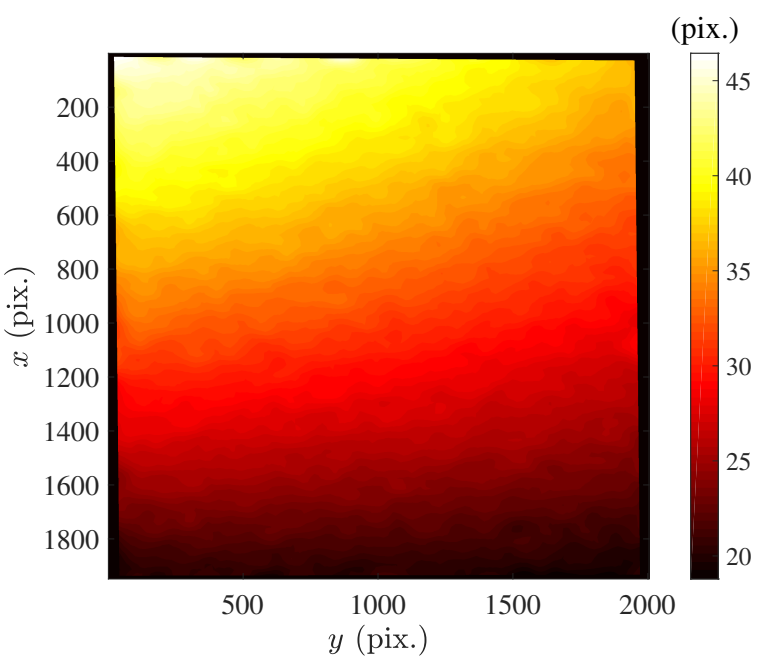

(d)

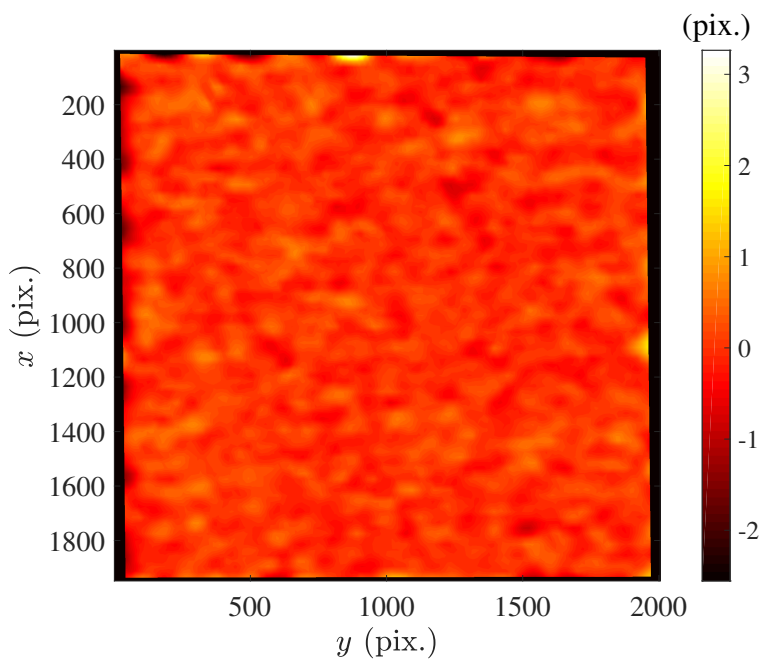

(f)

Figure 2: (a) SE image of horizontal Ge sample with Pt spots. (b) SE image of the Ge sample inclined at $70^{\circ}$ after applying tilt corrections. Displacement field as measured via DIC in $x$ (c) and $y$ (d) directions, respectively. Displacement field after removing global quadratic trend in $x$ (e) and $y$ (f) directions, respectively. The displacement is expressed in pixels and the pixel size is 53.7 $\mathrm{nm}$

According to Section 2.1. $u_{x}$ contains the topography information, which is vanishing in the present case, and possibly a linear component that is related to the inclination angle $\theta$. However, a long wavelength bias 
is seen in both $u_{x}$ and $u_{y}$ components. This is due to the scan artifacts that are not corrected. A second order polynomial regression is thus removed from the measured displacement as this bias is not considered as reliable, and being a low frequency modulation, it will lead to a minor change in the estimated topography. The resulting displacement components are shown in Figures 2(e) and 2(f). Contrary to the rather uniform $u_{y}$ field, the $u_{x}$ field exhibits horizontal bands. This is interpreted as a slow-scan direction error (due to the repositioning inaccuracy at the beginning of each new scanned line). An adapted global DIC procedure has been developed to correct this specific error by acquiring two images with different scanning directions [30, 31], yet the algorithm cannot be applied to two images on the inclined sample by the current SEM. Without correcting the slow-scan direction error, the root mean square (RMS) average of $u_{x}$ shown in Figure 2(e) is 0.41 pixel (or $22.3 \mathrm{~nm}$ ). The corresponding RMS average of topography measurement uncertainty is estimated to be $8.1 \mathrm{~nm}$ $\left(22.3 \mathrm{~nm} / \tan \left(70^{\circ}\right)\right)$. For comparison purposes, the RMS average of $u_{y}$ shown in Figure 2(f) is 0.23 pixel (or $12.3 \mathrm{~nm}$ ). The effect of the SEM scan drift distortion on the topography measurement is thus quantified.

It should be noted that an uncertainty level of $8.1 \mathrm{~nm}$ is very satisfactory, with respect to the pixel size of $53.7 \mathrm{~nm}$. Although the principles of tilt-imaging are far from new, the recourse to such high tilt angle is unusual, and global DIC used herein together with a well-suited speckle patterning provide very robust and accurate topography measurements.

\subsection{Test case}

The tensile test sample described in Ref. [31] is used to validate the tilt-imaging method. The same Pt speckles described in Section 2.2 have been deposited onto the sample surface. In addition, using the same technique, a Pt rectangle was drawn around the speckled region to easily locate the region of interest (ROI). The "horizontal" SE image of the sample is shown in Figure 3 The image definition is $3072 \times 3072$ pixels and covers an area of $130 \times 130 \mathrm{\mu m}^{2}$. The images have been acquired with an acceleration voltage of $30 \mathrm{kV}$ and a working distance of $13 \mathrm{~mm}$. To reduce the possible SEM drift distortion, each SEM image is the average of 30 scanned frames. The dwell time has been set to 1 s to increase the image acquisition speed. With these settings, 5 minutes were needed per acquisition.

The SEM drift distortion for the aforementioned conditions have been analyzed in Ref. [31], and amount to approximately $1 \%$ in both $x$ and $y$ directions. The uniform linear SEM drift distortion will not lead to topography measurement errors, since its effect will be removed in the linear fitting step. The random SEM drift from line to line has been analyzed and quantified in Section 2.2. As a result, the large image resolution together with the adopted acquisition conditions provide a rich characterization of the sample surface, while the uncertainty due to SEM drift is kept to a limited level. 


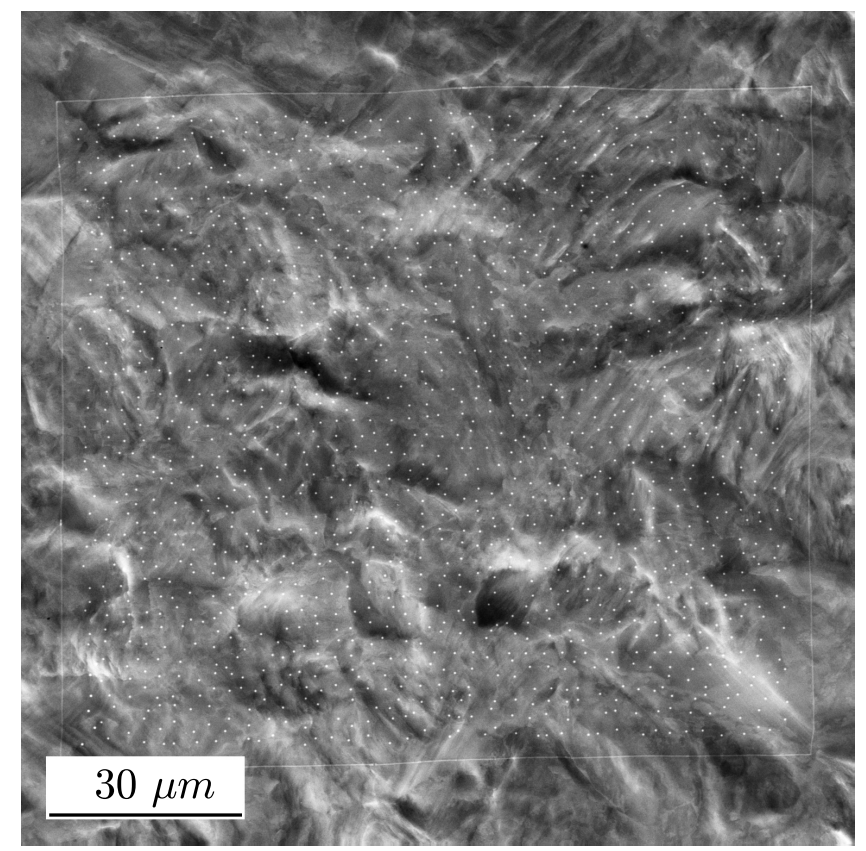

Figure 3: SE image of the sample in the horizontal configuration.

Then the sample is tilted by about $70^{\circ}$ and an $\mathrm{SE}$ image of the same size is recorded without activating the tilt correction function of the SEM (Figure 4(a)). The stage rotation parameter of the SEM has been adjusted to make the Pt rectangle horizontal. The lateral surface of the sample is imaged in the bottom part of the image. The "valleys" and "hills" are very prominent on the sample surface because of the shadowing effect of a rather grazing incidence. The affinity due to tilt along the vertical direction is first corrected, as shown in Figure 4(b), by a scale factor of $1 / \cos (\theta)=2.7$, where this value is a rough estimate obtained by tracking the rectangular marking on the surface. This factor will however be accurately determined in the following and hence this first rough determination is only aimed at initializing the registration procedure. 


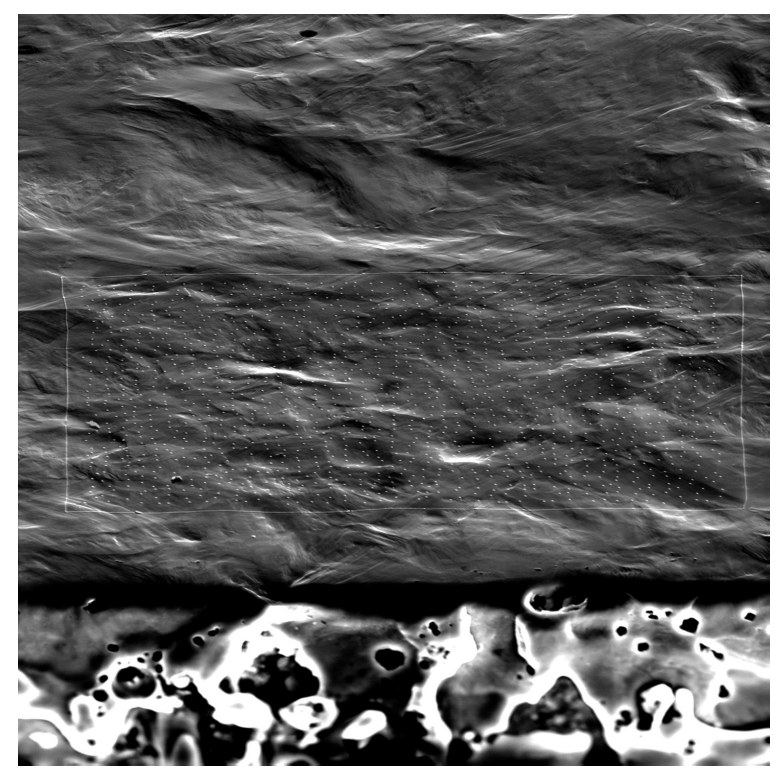

(a)

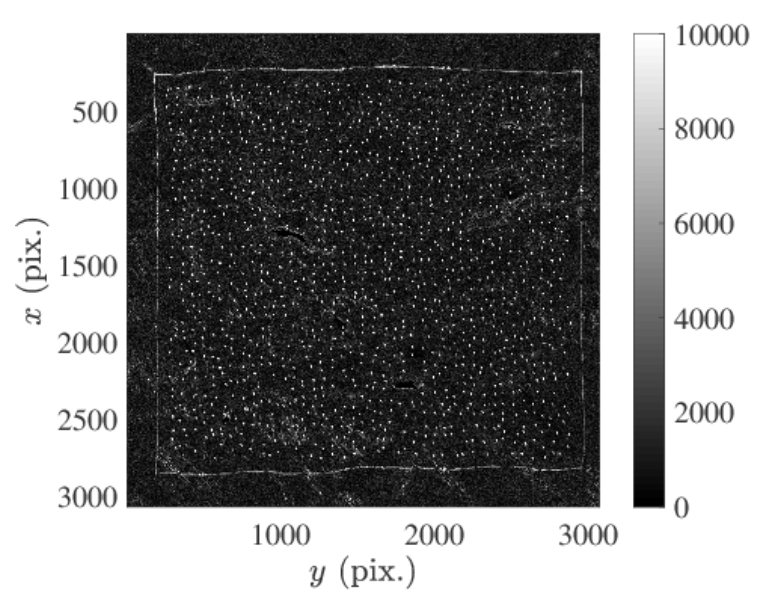

(c)

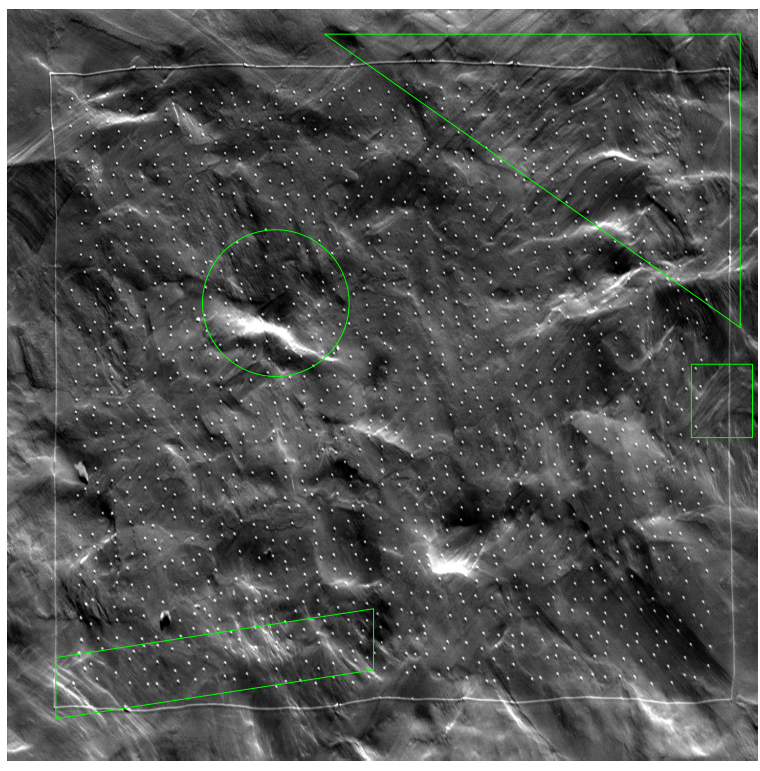

(b)

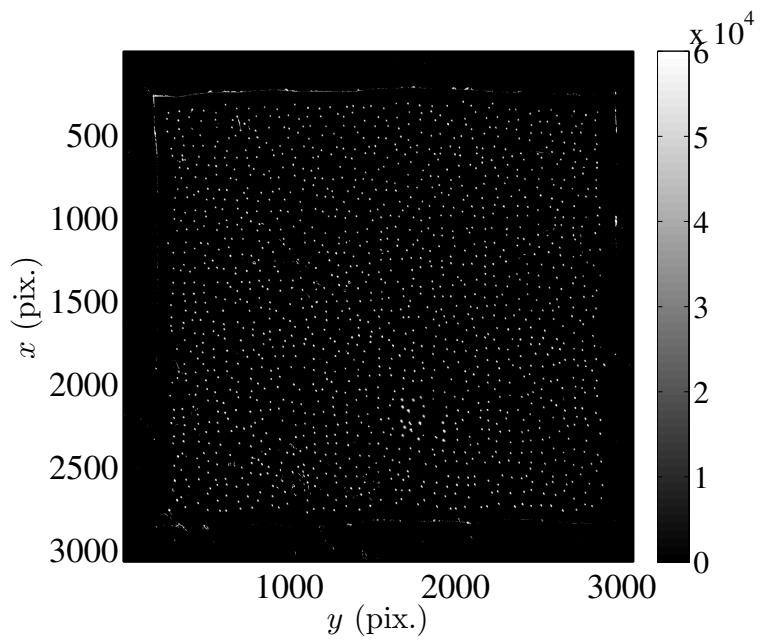

(d)

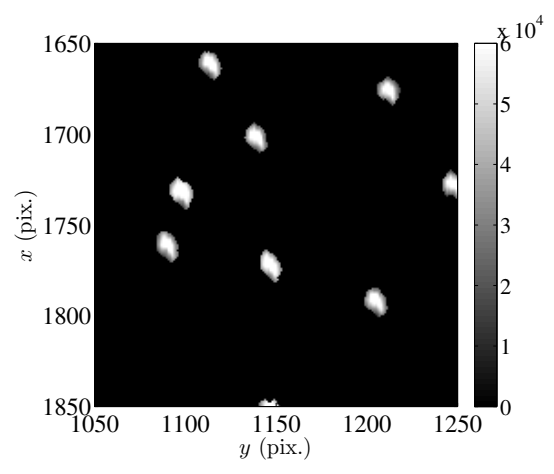

(e)

Figure 4: Pre-correction of the tilted image. (a) Original SE image of the tilted sample. (b) Stretched image 2.7 times along the vertical direction. Several regions with visible slip traces are indicated in green. (c) Norm of the gray level gradient. (d) Processed tilted image and (e) zoom to show the rendering of the Pt spots. The pixel size is $42 \mathrm{~nm}$ for all images.

In such a transformation, the brightness and contrast change very significantly, which is due to the interaction between the e-beam and the sample surface. The goal is not to explore such physics and propose a model interpreting the gray levels. Rather it is preferred to focus on the surface marking, namely, the Pt spots that 
were deposited as a speckle pattern. They are very clearly visible in the reference image. They become also much clearly visible in the tilted image (Figure 4(a) and 4(b) . In order to filter out the brightness information of the substrate material, the norm of the gray level gradients is calculated (Figure 4(c)]. The Pt spots are better revealed, which is due to their high gray level variations. A threshold has been applied to Figure 4(c) to get the positions of all the Pt spots, with the exception of a few locations that cannot be distinguished from the bright background. The gray level of Pt spots and their vicinity is kept, while all other pixel levels are set to 0 in the treated tilted image (Figure 4(d)]. A zoom of the final image is shown in Figure 4(e) where the gray level gradient at $\mathrm{Pt}$ spots are visible.

The displacement field is assessed via DIC between the two images shown in Figure 3 and 4(d). A finiteelement description of the kinematics is used with 3-noded elements with linear interpolation functions (i.e., T3-DIC [32]). Only the region covered by Pt speckles is studied (Figure 5(a) , and an unstructured mesh with characteristic length of 25 pixels is used.

Due to the concentration of contrast around Pt speckles (Figure 4(d)], regularization is introduced to provide a smooth interpolation at very small scales when Pt speckles are not seen or very faintly. A regularization based on an elastic kernel [32] is utilized in the present work to filter out high frequencies, with a cut-off frequency (i.e., the inverse of the so-called regularization length) that can be tuned at will. The regularization length has been gradually reduced from 400 pixels down to 100 pixels. It can be seen in the DIC residual field (i.e., the difference between reference and corrected deformed images after registration) shown in Figure 5(b) that all the Pt speckles are well matched between both images, with the exception of the lost speckles in bright regions shown in Figure 4(b), Displacement fields in $x$ and $y$ directions are shown in Figure 5(c) and 5(d). 


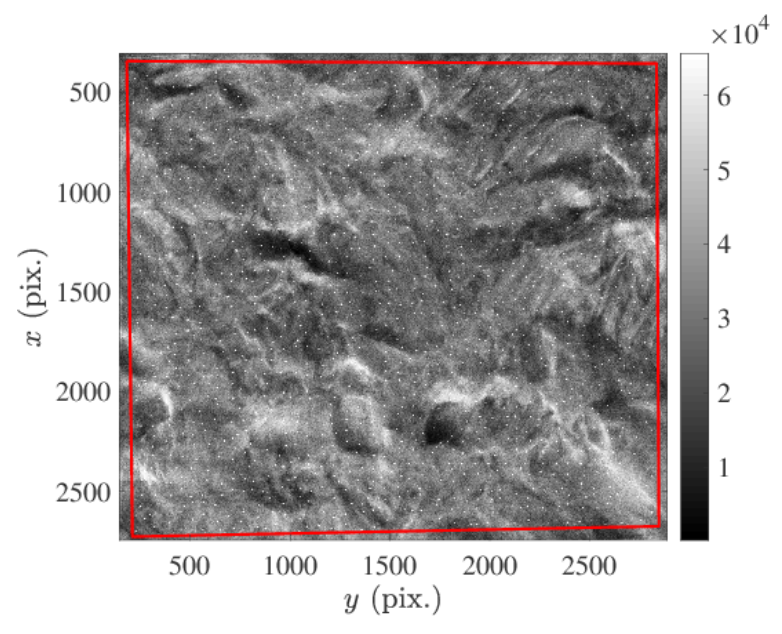

(a)

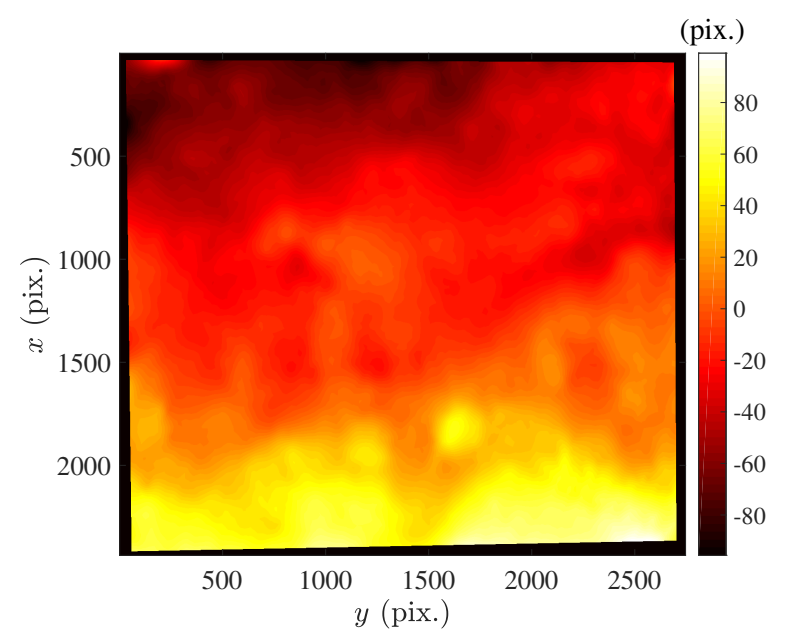

(c)

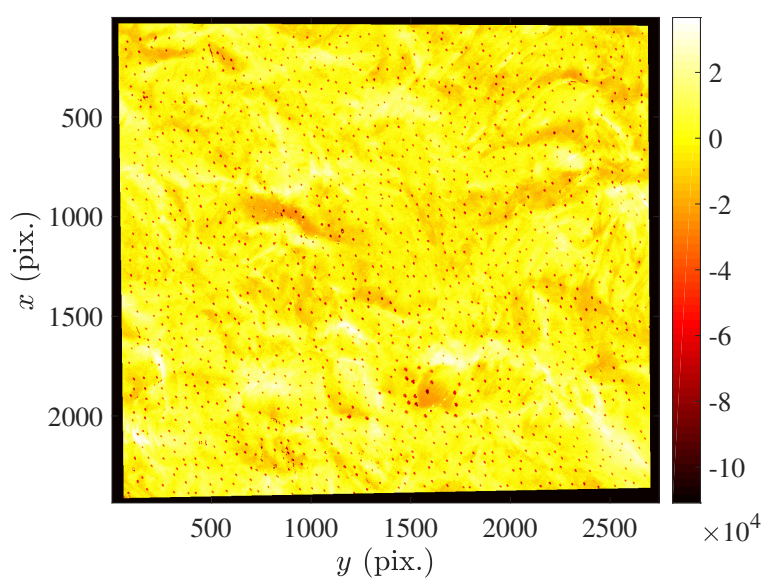

(b)

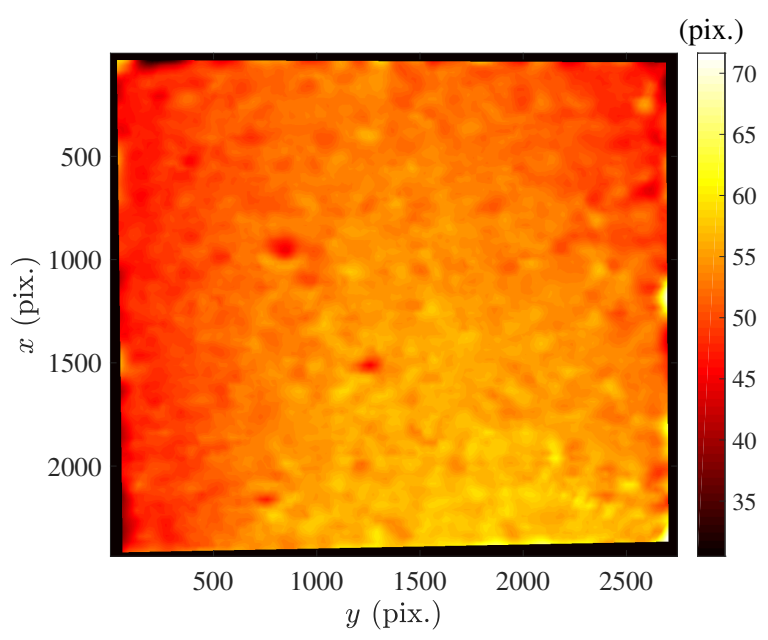

(d)

Figure 5: DIC calculation in the tilt-imaging method. (a) Region of interest for DIC. (b) Gray level residual field. (c,d) respectively $x$ and $y$ component of the resulting displacement field (expressed in pixels, 1 pixel $\leftrightarrow 42 \mathrm{~nm}$ ) between the images shown in Figures 3 and $4(\mathrm{~b})$

The $u_{x}$ displacement component shows a significant gradient along $x$, proving that the initial guess used for the pre-correction by the scale factor $1 / \cos (\theta)=2.7$ was not the best suited value. A linear regression through the data is performed to perform a better tilt compensation, leading to a much more precise estimate of the tilt angle

$$
\cos (\theta)=0.39
$$

or $\theta \approx 67.1^{\circ}$. It should be noted that a precise assessment of the tilt angle in EBSD positions has a big impact on EBSD acquisitions, not only on the absolute value of the indexed crystallographic orientation, but also on the coordination of EBSD figures after the tilt correction [33. It is believed that this method could become a routine process prior to EBSD acquisition when high precision is required.

Then the topography field $h$ is computed from $h(x, y)=u_{x} \cot (\theta)$ (see Figure 6(a). For comparison purposes, the ferritic grains in the studied region are manually selected and highlighted in Figure 6(b). The selection criterion of ferritic grains is the absence of lath structure in the range 1-3 $\mu \mathrm{m}$. 


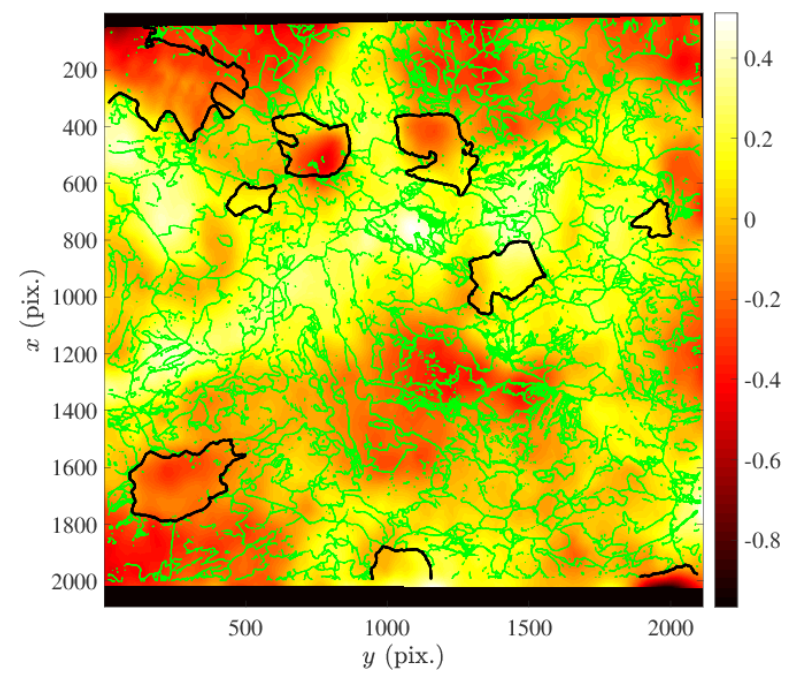

(a)

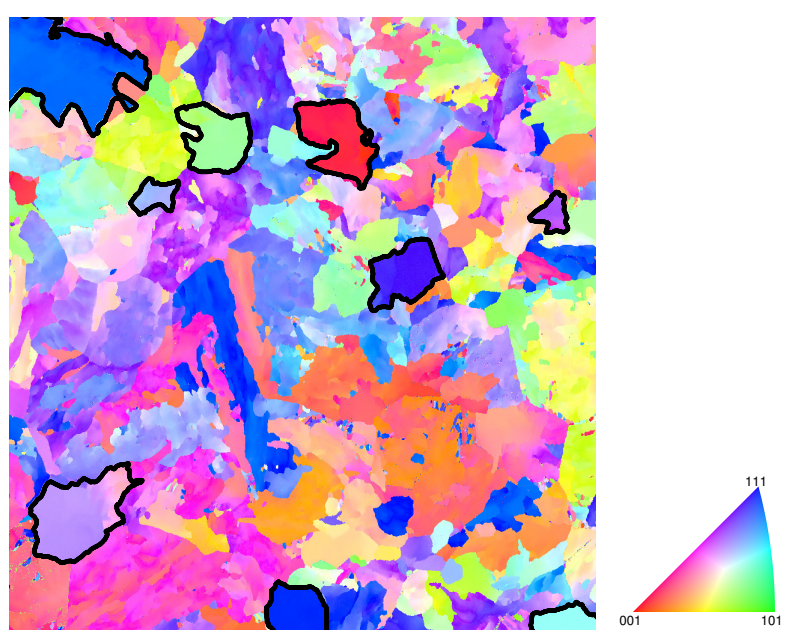

(b)

Figure 6: (a) Estimated topography field. To better illustrate the relationship between topography and sample microstructure, the grain boundaries are shown as green lines for bainite and black lines for ferrite. (b) Ferritic grains in the studied region are highlighted for comparison purposes. The pixel size is $50 \mathrm{~nm}$.

The RMS average of the topography measured by tilt-imaging is $188 \mathrm{~nm}$, while the maximum peak-to-value distance is $1.36 \mathrm{\mu m}$. It is concluded from Figure 6 that the height of large ferritic grains tends to be lower than average, especially at the grain center. This phenomenon is not surprising, as this softer phase tends to elongate more in a tensile test, thus due to plastic incompressibility, the surface contracts more than other grains. Note that there exist several regions rich of visible activated slip systems in Figure 4(b), which indicate the presence of "paddy-rice-field"-like topography. However, these structures are beyond the resolution and thus not captured in Figure 6(a). A higher resolution and suited marking would be required to resolve them.

The sizes of Pt speckles are adjusted for EBSD acquisitions. Consequently, relatively large and rather few spots have been deposited onto the sample surface. If topography were chosen to be the principal quantity of interest, then a finer and denser speckle marking would lead to a better spatial resolution. In the present case, the evaluation of topography was seen as an opportunity, namely, a side-result that could be obtained at no additional cost after an EBSD analysis was chosen.

It should be emphasized that the tilt-imaging method does not differ from standard stereoscopic SEM techniques in terms of underlying principle. However the stereoscopic technique usually involves two acquisitions with a tilt difference of about $10^{\circ}$. Thus the displacement magnitude between the acquired images is very limited. By adopting a fixed detector, in-lens or not, and tilting the sample, the "stereo-angle" can be chosen freely, preferably larger tilt angles to increase the signal-to-noise ratio. As soon as an EBSD device is installed in an SEM, such analysis can be performed by acquiring an additional SE/BSE image (without the tilt correction functionality), provided sufficient surface markings are engineered to enable for image registration. Further, as the above result shows, regularized DIC makes use of the limited and localized contrasted areas between the two stereo-images to measure a displacement field, thereby resulting in a smooth topography for the whole area of interest. The abrupt slope change at mesh boundaries is thus avoided.

\section{FIB method}

After the in situ tensile test, a region of the same sample has been chosen as potential FIB milling area. This region, roughly $1 / 9$ th of the speckled part of the sample surface, is located near the edge to make milling easier and at the boundary middle of the speckled zone to benefit from precise displacement measurements. 
The whole area around the ROI is FIB-milled with high current to free the access and limit pollution during further analyses. This preparatory step can also reduce the volume to be milled during the main FIB-EBSD process, thus accelerate the experiment and reduce the potential coordinate drift. The ROI has been covered by a $1 \mu \mathrm{m}$-thick layer of Pt (Figure 7(a). This Pt coating acts as a sacrificial protective layer for FIB milling. Moreover, performing EBSD after successive slice removals, amorphous Pt is easy to distinguish from the steel substrate. The successive interfaces with Pt follow precisely the initial free surface of the sample. Note that a similar protective tungsten layer was deposited in the past to mainly protect the free edges against diffuse FIB cutting [13. So-called 3D-EBSD provides a much richer information than surface topography, but this technique also allows for quantitative measurements of topography, and is used herein as the ground truth.

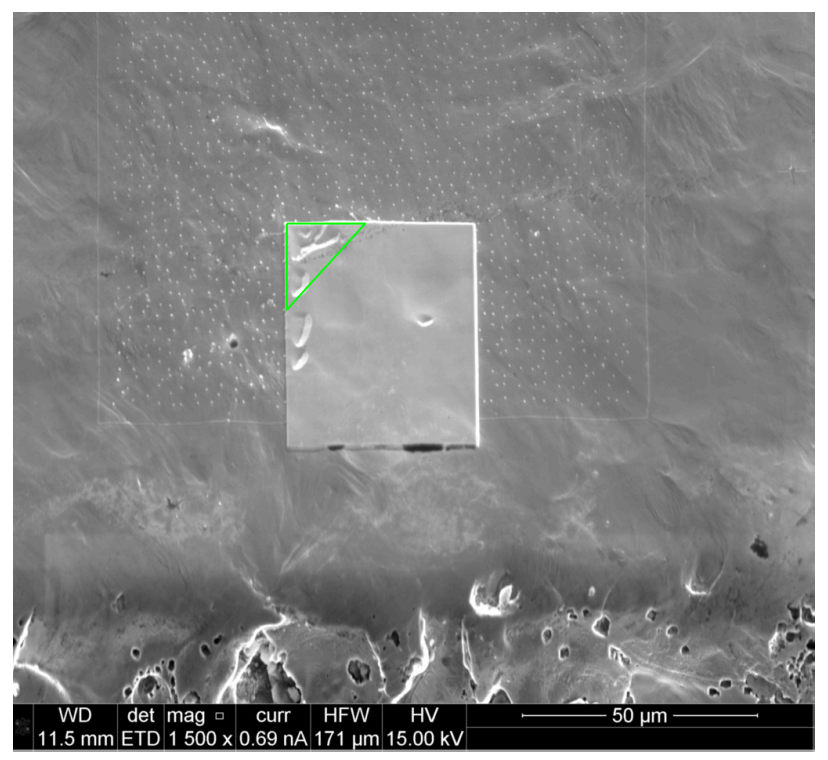

(a)

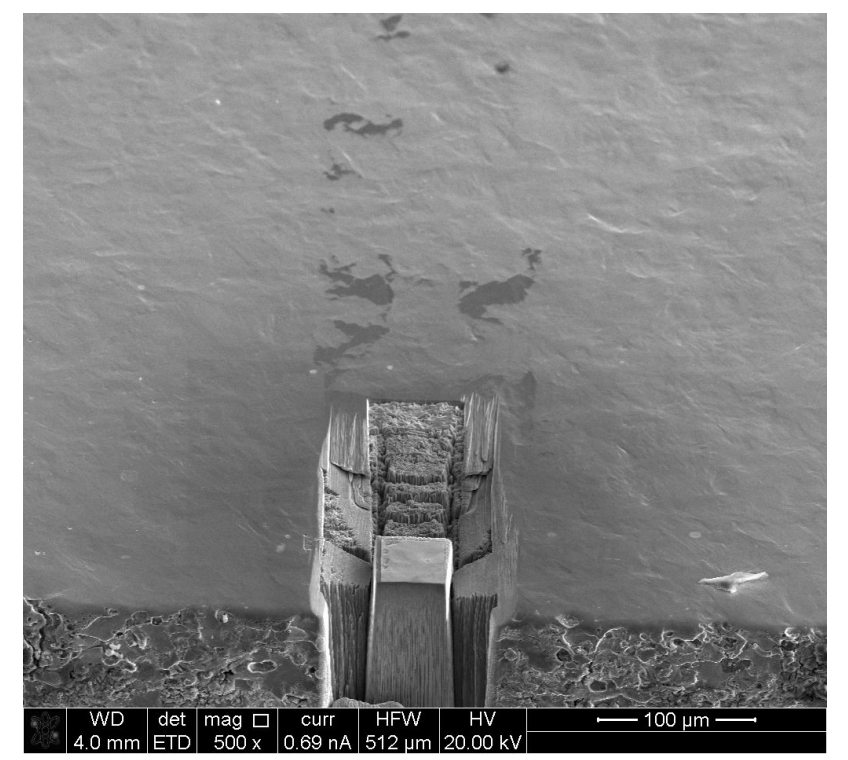

(b)

Figure 7: Preparations of FIB-EBSD. (a) Chosen area covered by a layer of Pt. (b) Neighborhood of the area of interest is first removed to make milling easier. The volume to follow the 3D-EBSD procedure is at the top of the pillar that was carved out (bottom of sub-figure (b)).

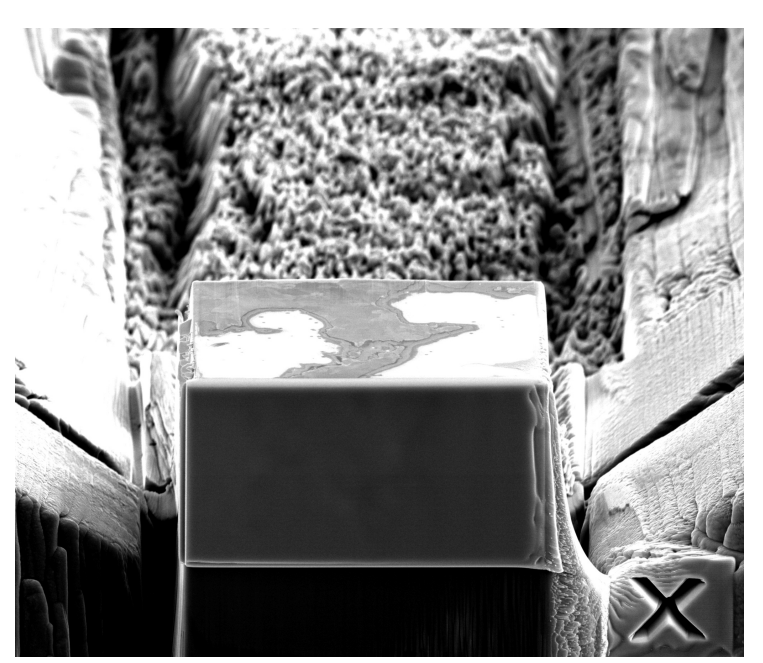

(a)

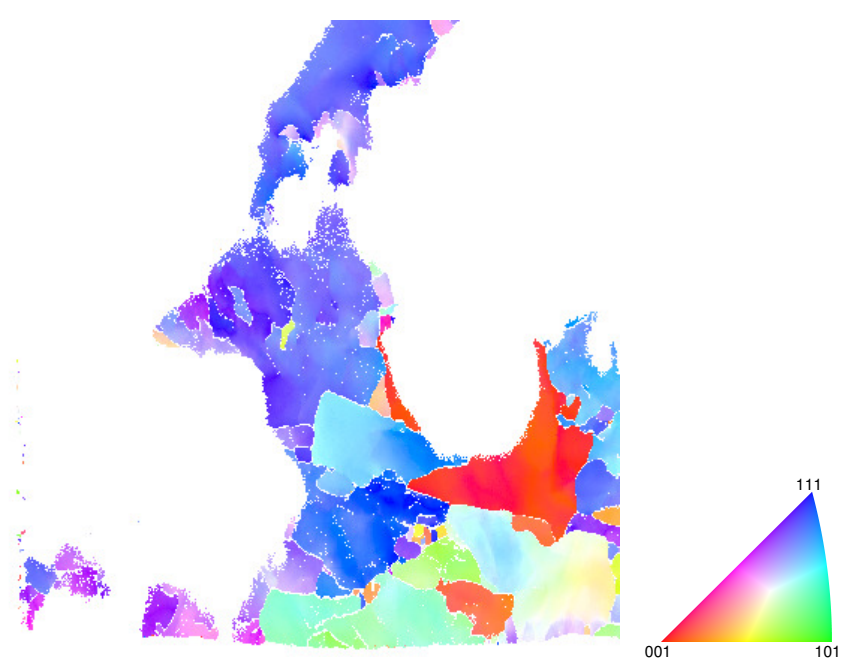

(b)

Figure 8: (a) Sample aligned in FIB position at an intermediate stage of the 3D-EBSD procedure. The top of the pillar shows both the emergence of the most salient regions of the sample surface and the Pt covered deeper regions. (b) Indexing of crystal orientation by EBSD at the same stage. 
Prior to the main FIB-EBSD process, the surface of the ROI has FIB-milled with high current to ensure a flat surface thus prevent "theater curtaining" 34]. Special care is required to enable for accurate $z$-direction realignment after each sample motion as FIB milling and EBSD analyses require different positions of the sample in the SEM chamber. A cross-shaped mark has been milled close to the studied area for such an alignment. The software EBS3 G2 used for FIB-EBSD recognizes the mark and measures its motion by cross-correlation with sub-pixel resolution. The sample in the FIB position is shown in Figure 8(a) after few milling steps. The clear contrast at the sample surface reveals that the substrate material emerges at some places while the Pt coating persists at others. The crystal orientation map as indexed by EBSD (Figure 8(b) accurately quantifies this analysis. The acceleration voltage has been $30 \mathrm{kV}$, and the physical size of one pixel $100 \mathrm{~nm}$ for every EBSD acquisition. Each EBSD indexing takes about $50 \mathrm{~min}$. The pollution induced by the EBSD analysis has been eliminated by the next FIB milling step.

In total, 118 slices have been milled and indexed for a duration of one week. A volume of $42 \times 41 \times 11.8 \mu^{3}$ is available. The orientation maps of the first eight slices of FIB-EBSD are shown in Figure 9 . The white area indicates the not-indexed region, which is covered by the Pt layer. As a result, the first 8 inverse pole figures can be seen as a binary height image for sample surface. The Pt layer gradually recedes and completely disappears at the eighth slice. The first slice shown in Figure 9(a) has already a significant indexed area, especially at the upper left region. This is where the Pt deposition is thinner, as shown in the boxed region of Figure 7(a) Besides, the FIB-milling of the ROI surface before FIB-EBSD (in order to remove the roughness of the ROI surface) could have removed the Pt layer, even some substrate material, at peaks of the sample. This phenomenon implies that the Pt layer was not thick enough and presumably the most salient peaks of the topography were erased during the first surface preparation. 


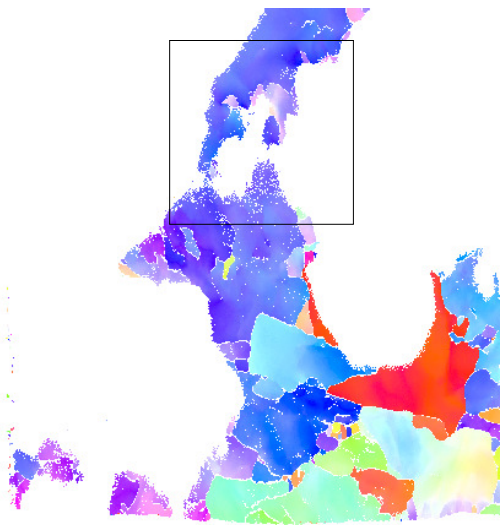

(a)

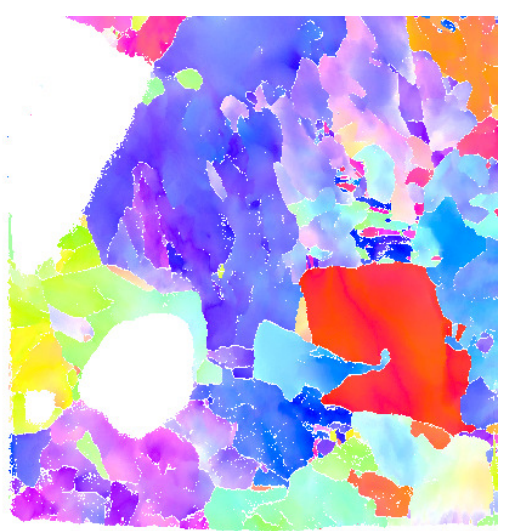

(d)

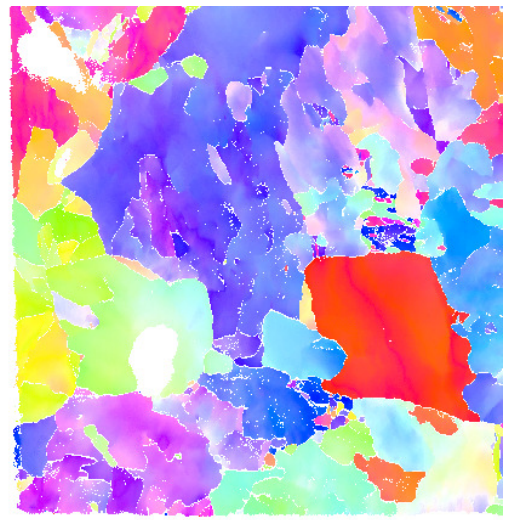

(g)

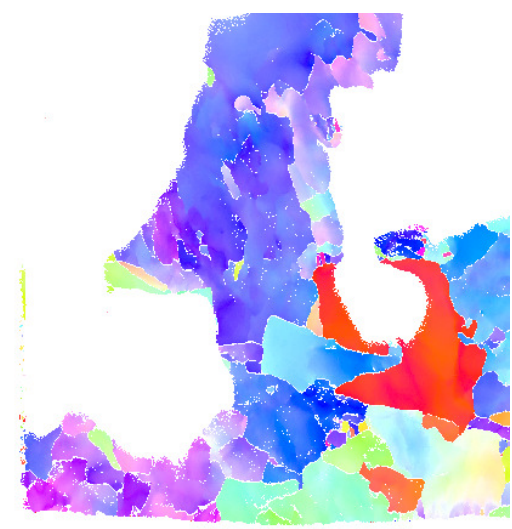

(b)

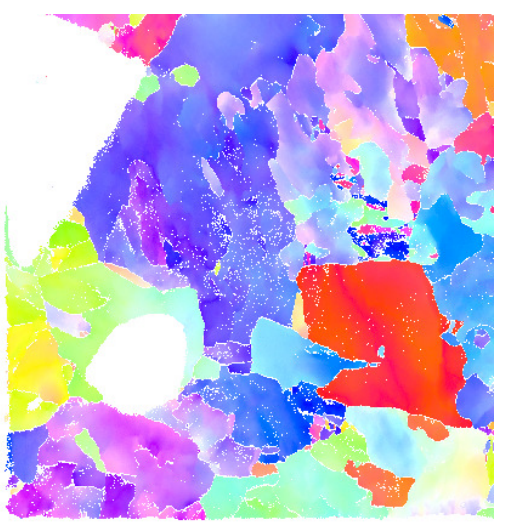

(e)

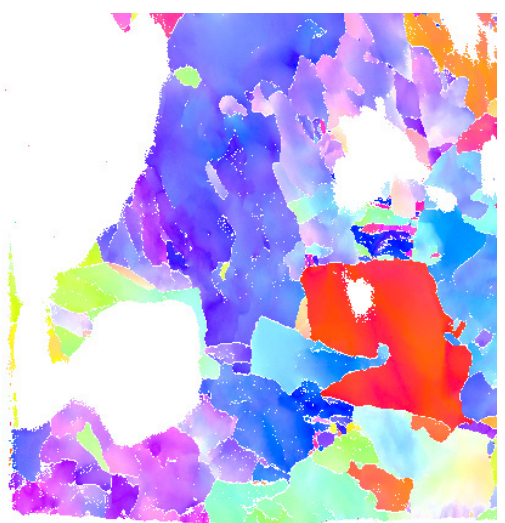

(c)

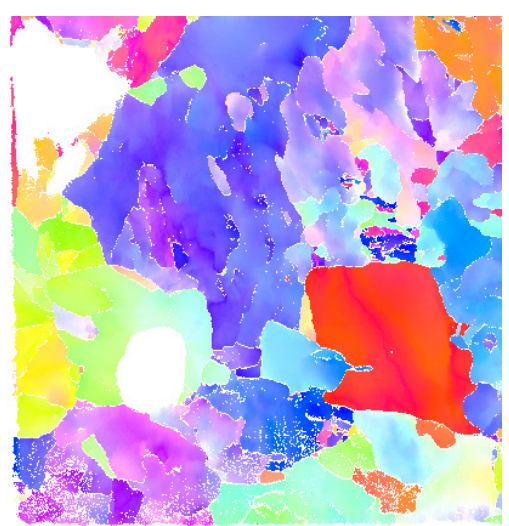

(f)
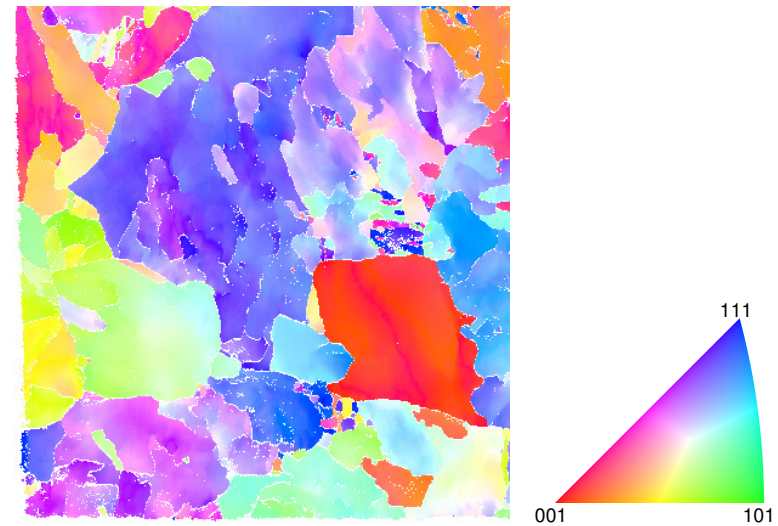

(h)

Figure 9: Orientation maps of the first 8 FIB-EBSD slices. The deposited Pt layer (appearing in white) is completely removed in the eighth slice.

The transition between indexed and not-indexed areas is gradual in most cases, thus the indexed grains near the remaining Pt layer seem more "transparent," as shown in the boxed area of Figure 9(a) A filter process is applied to provide a more clear-cut binary height image. Simple image processing operations are used, "open" and "close" steps involving only nearest neighbor pixels so as not to alter the large scale features by artificial smoothing. The effect of the filter is shown in Figure 10, It is observed that not only a more precise topography image results from such procedure, but also a finer microstructure is revealed. 


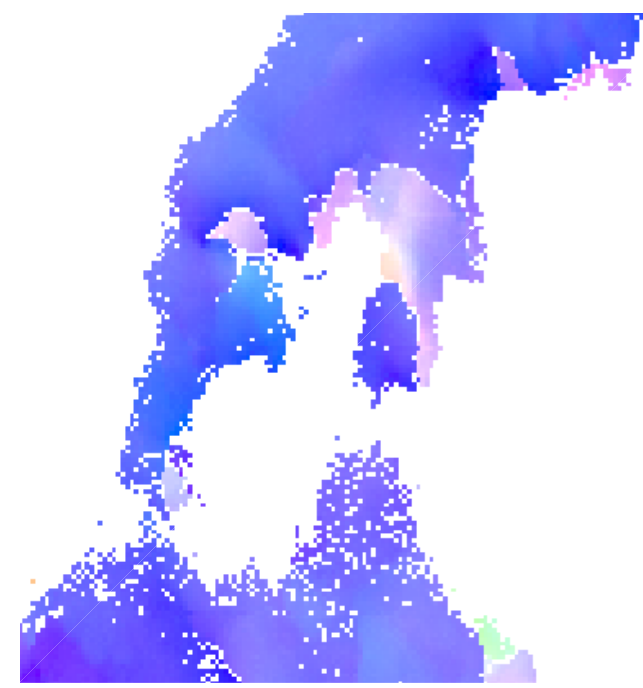

(a)
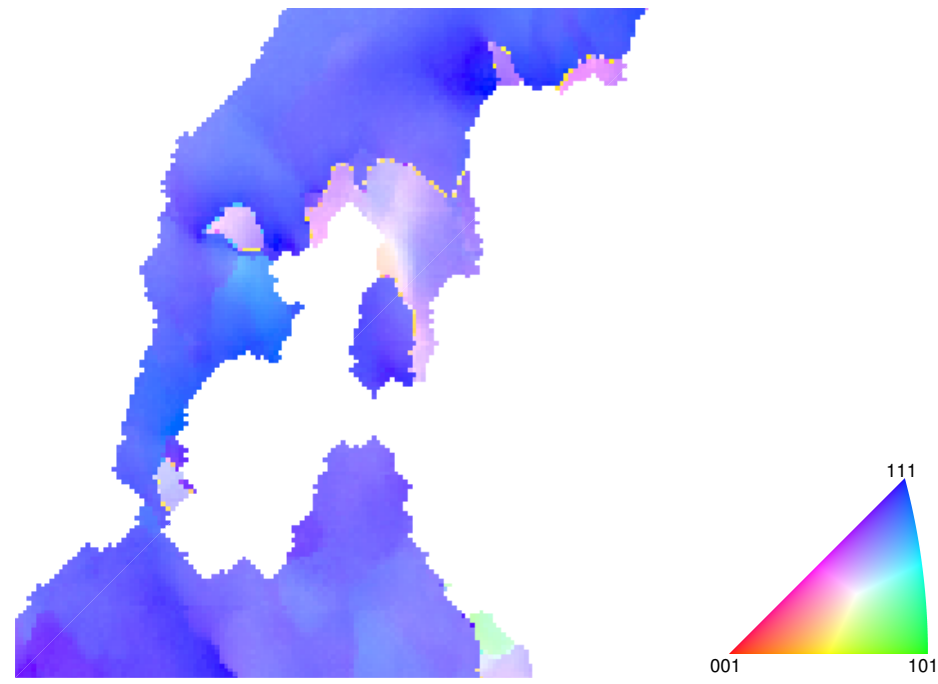

(b)

Figure 10: Effect of filter operation on FIB-EBSD images. (a) Raw FIB-EBSD figure extracted from Figure 9(a) (b) Filtered image. Image "open" and "close" steps, and interpolation of the crystallographic orientation are involved.

The filtered 3D-EBSD data were collected and processed with Dream3D, an open source software dedicated to the analysis of 3D microstructures [35]. For FIB-EBSD data, the code reads the indexed orientation maps, realigns each slice in the $(x, y)$ plane and labels each grain based on a user-defined disorientation threshold. Slice realignment is necessary since shift inevitably occurs between subsequent EBSD acquisitions.

Indexing of crystal orientation at grain boundaries is difficult and imprecise, and one should pay attention to automatic corrections performed on non-indexed pixels. Here, the Pt coating phase was labelled manually as a grain on its own to prevent spurious modifications of the Pt-sample interface in the standard data "cleaning" procedure that is automatically performed. After completion, it suffices to delete the Pt grains and the topography becomes visible (Figure 11(a)). Besides, a full crystallographic image of the sample surface is also obtained by combining the first 8 FIB-EBSD slices (Figure 11(c)). The reconstructed sample surface is identical to the inverse pole figure of the sample before Pt layer deposition, which can be found in Ref. 31. It should be noted that due to the discrete nature of the FIB milling process, the topography reconstructed by this procedure has terraces, each of height $100 \mathrm{~nm}$ (or 1 pixel). To filter out the topography while preserving the terrace edges, successive Fourier filtering enforcing the terrace edges has been performed, and the result is shown in Figure $11(\mathrm{~b})$. 


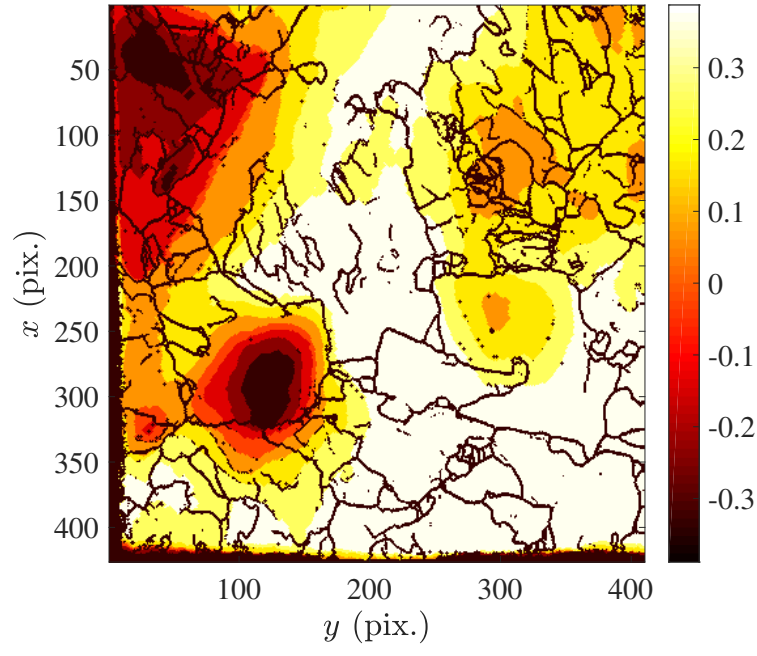

(a)

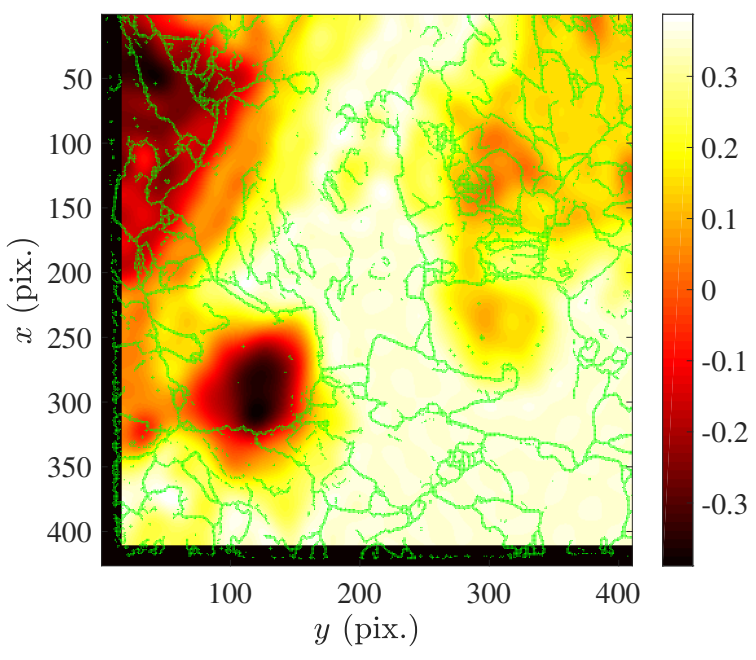

(b)
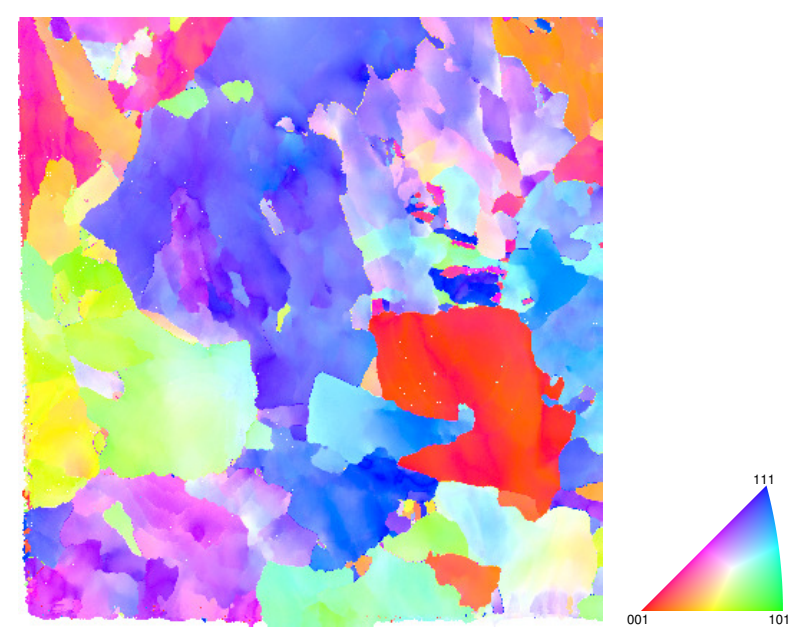

(c)

Figure 11: Reconstructed sample surface by FIB-ESBD. (a) Raw topography (expressed in $\mu m$ ). (b) Topography with a Fourier transform interpolation based on on-edge pixels. (c) Inverse pole figure of the sample surface obtained from the first 8 slices of 3D-EBSD procedure. The pixel size is $100 \mathrm{~nm}$ for all images.

\section{Rotation-integration method}

\subsection{Mechanisms}

Quaternion correlation provides the displacement field and, more importantly, the crystal rotations [27. With the exception of plastic strains due to the motion of dislocations along activated slip systems, the displacements in all three directions are related to crystal rotations. This principle can be used to extract the fraction of topographic changes due to plastic flow and due to crystal rotation.

In the following to make the discussion easier, it is first assumed that no plastic flow has occurred. The crystal rotation, which is applied to the pixel length, is then interpreted as the antisymmetric part of the displacement gradient (within the small perturbation framework). Moreover the sample surface is traction free, and hence the symmetric part of the displacement gradient (i.e., the infinitesimal strain tensor $\boldsymbol{\epsilon}$ ) is constrained. In particular, vanishing shear stresses and strains $\varepsilon_{x z}=\varepsilon_{y z}=0$ imply that the change in topography, $u_{z}$, which is the change in surface elevation between the two states chosen for quaternion correlation, are related to the 
rotation matrix $[\boldsymbol{Q}]$ based on axis-angle $\left(\omega,\left[\Omega_{x}, \Omega_{y}, \Omega_{z}\right]\right)$

$$
[\boldsymbol{Q}]=\left[\begin{array}{ccc}
\left(1-\Omega_{x}^{2}\right) \cos \omega+\Omega_{x}^{2} & \Omega_{x} \Omega_{y}(1-\cos \omega)+\Omega_{z} \sin \omega & \Omega_{x} \Omega_{z}(1-\cos \omega)-\Omega_{y} \sin \omega \\
\Omega_{x} \Omega_{y}(1-\cos \omega)-\Omega_{z} \sin \omega & \left(1-\Omega_{y}^{2}\right) \cos \omega+\Omega_{y}^{2} & \Omega_{y} \Omega_{z}(1-\cos \omega)+\Omega_{x} \sin \omega \\
\Omega_{x} \Omega_{z}(1-\cos \omega)+\Omega_{y} \sin \omega & \Omega_{y} \Omega_{z}(1-\cos \omega)-\Omega_{x} \sin \omega & \left(1-\Omega_{z}^{2}\right) \cos \omega+\Omega_{z}^{2}
\end{array}\right]
$$

The calculation of $\left(\omega,\left[\Omega_{x}, \Omega_{y}, \Omega_{z}\right]\right)$ from reference and deformed EBSD images is detailed in Ref. [27]. From this rotation field, the initially flat surface roughens and the topography gradients read

$$
\begin{aligned}
G_{x} & =e_{z} \cdot Q \cdot e_{x} \\
G_{y} & =e_{z} \cdot Q \cdot e_{y}
\end{aligned}
$$

where $\boldsymbol{e}_{i}$ is a unit vector in direction $i$ (i.e., $x, y$ or $z$ ), and

$$
\begin{aligned}
G_{x} & =u_{z, x} \\
G_{y} & =u_{z, y}
\end{aligned}
$$

Note that the same argument would also hold for finite strains using the polar decomposition of the deformation gradient tensor.

The two fields $G_{x}$ and $G_{y}$ are theoretically related by the equality of cross-derivatives $G_{x, y}=G_{y, x}$. However, since they are estimated independently, numerically this relationship is violated (due to measurement noise). In order to minimize the effect of data noise, an integration using gradients in both directions via Fourier transform is well suited [36]. Denoting Fourier transforms with a ${ }^{\sim}$ symbol, and wave vectors as $\boldsymbol{k}$, the change in elevation $u_{z}$ reads

$$
\widetilde{u_{z}}=\frac{-i}{2 \pi|\boldsymbol{k}|^{2}}\left(k_{x} \widetilde{G_{x}}+k_{y} \widetilde{G_{y}}\right)
$$

which means that $u_{z}$ variations are calculated from crystal rotations.

\subsection{Uncertainty assessment}

Before applying the rotation-integration method to the real topography, an uncertainty evaluation is performed. Two successive EBSD acquisitions have been performed on the initial (i.e., before tension) 16MND5 steel sample with a polished surface. The first image, shown in Figure 12(a), is arbitrarily chosen as the reference state and the second as the "deformed" configuration (Figure 12(b)]. Quaternion correlation on these two images provides the "crystal rotation" between them, which combines the systematic and random errors in the crystallographic orientation determination of the SEM. Figure 12(c) shows the misorientation magnitude, $\omega$, and Figures $12(\mathrm{~d})$, $12(\mathrm{e})$ and $12(\mathrm{f})$ display the three components of a unit vector $\Omega$ along the rotation axis. The mean value of $\omega$ is $0.69^{\circ}$, and its standard deviation is equal to $0.39^{\circ}$. 


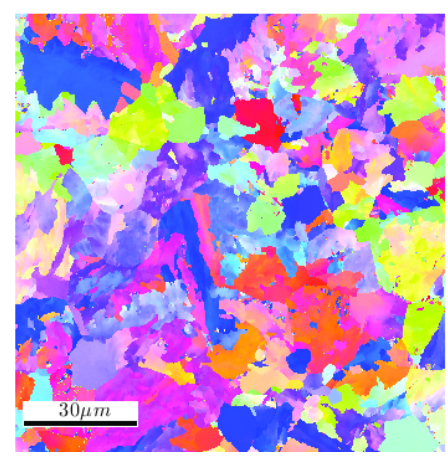

(a)

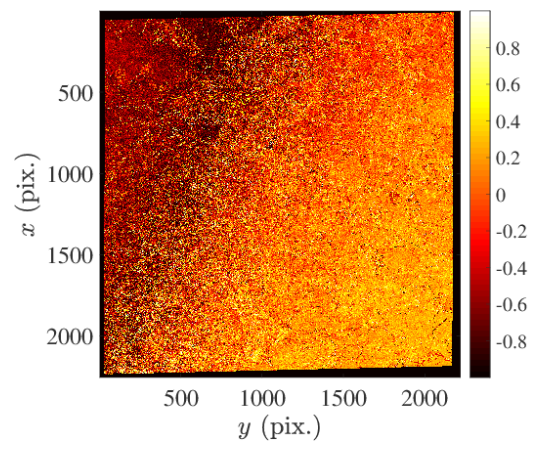

(d) $\Omega_{x}$

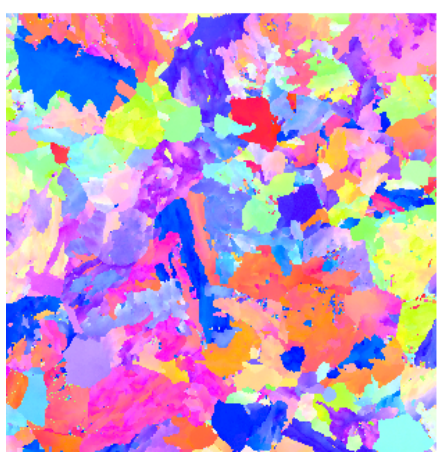

(b)

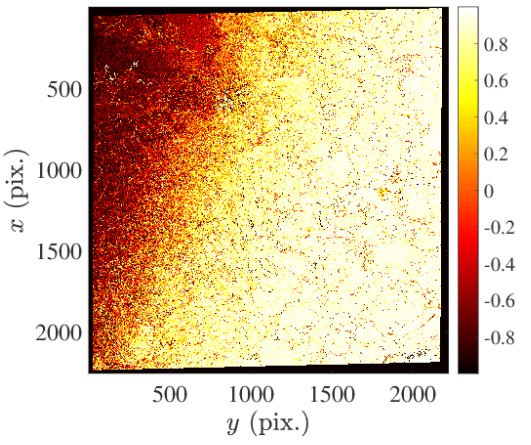

(e) $\Omega_{y}$

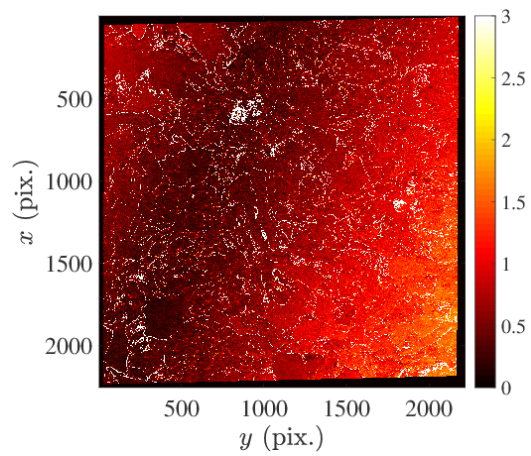

(c) $\omega$

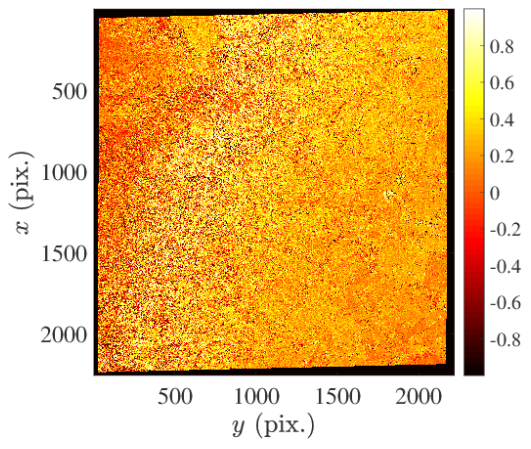

(f) $\Omega_{z}$

Figure 12: Reference (a) and "deformed" (b) states of the polished sample surface. (c) Rotation angle $\omega$ (in degrees). (d-f) $x, y$ and $z$ components of the $\boldsymbol{\Omega}$ rotation axis. The pixel size is $50 \mathrm{~nm}$ for all images.

The corresponding topography gradients in $x$ and $y$ directions are shown in Figure 13(a) and 13(b). Combined with Figure 12. low frequency fields are seen especially in the $G_{y}$ component. Because the initial geometry is flat, the displacement along $z$ is equivalent to the elevation. When integrated, the topography is obtained (Figure 13(c)]. Rigid body motions of the sample between the two images can neither explain the misorientation shown in Figure 12(c), nor the integrated topography shown in Figure 13(c), This significant long wavelength misorientation could be attributed to the inadequate precision of pattern center during orientation indexing [37. A 2nd order polynomial regression through the elevation map is performed and removed in Figure 13(d). Compared to Figure 13(c), the 2nd order polynomial removes the majority of spurious topography, while the resulting elevations in Figures 13(d) and 13(e) imply that a 4th order polynomial is necessary to remove the remaining signal (Figure 13(f)]. However, a regression by higher order polynomials may delete real signals, thus a compromise has to be made in real measurement cases. It is concluded that, after 4th order polynomial regression, the standard uncertainty of topography measurement is about $10 \mathrm{~nm}$. 


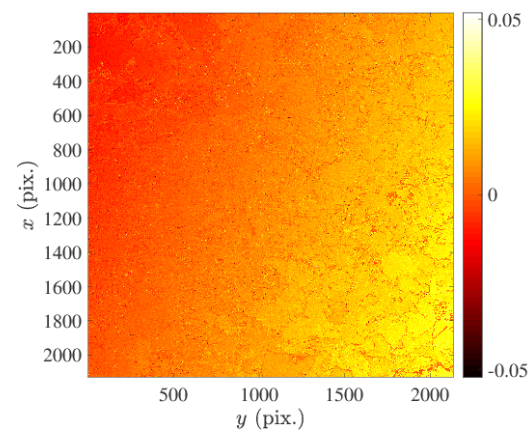

(a)

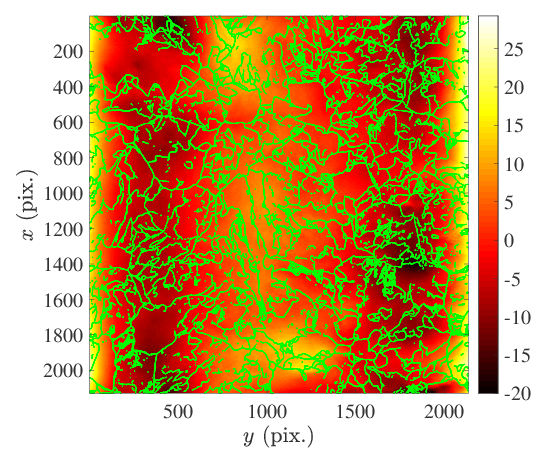

(d)

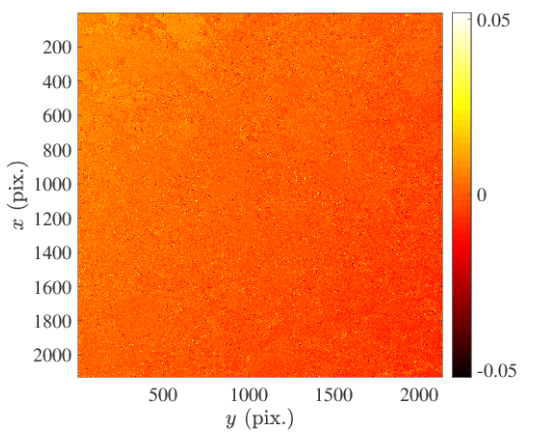

(b)

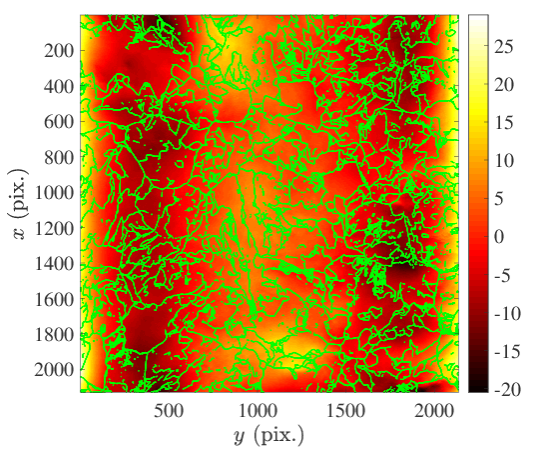

(e)

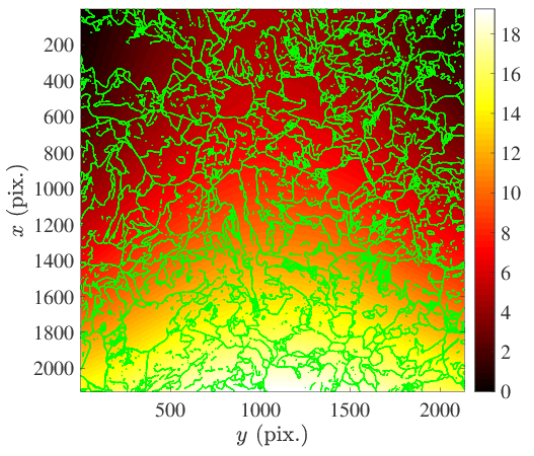

(c)

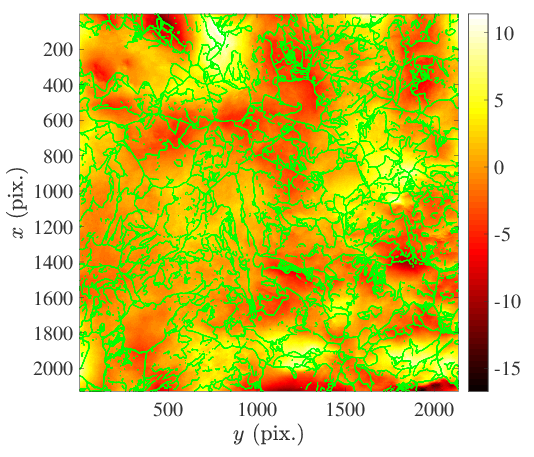

(f)

Figure 13: Surface elevation gradient in (a) $x$ and (b) $y$ directions obtained by crystal rotation. (c) Integrated elevation (expressed in $\mu \mathrm{m}$ ) from the gradients. Elevation (expressed in $\mathrm{nm}$ ) after removal of quadratic (d), 3rd-order (e), or 4th-order (f) polynomial trends. The pixel size is $50 \mathrm{~nm}$ for all images.

The method was also applied to two successive EBSD acquisitions on the deformed sample surface to measure the topography reconstruction uncertainty. Again the artifactual topography due to global orientation drift is assumed to be removed with a 4th-order polynomial. The remaining topography field has a standard uncertainty of $11 \mathrm{~nm}$, slightly higher than the previous test, due to the degraded EBSD image quality on strained samples. Consequently, it is observed that the SEM used in the present work has a non-negligible orientation indexing "drift," which makes the removal of fitted 4th-order polynomials from the topography necessary. As a result, only the local relative topography remains after this removal and is thus accessible for the EBSD images acquired by the tested SEM machine.

\subsection{Analysis of the test}

The EBSD acquisitions are first rectified to correct the coordinate distortions [31, then analyzed by the rotation-integration method. Figure 14(a) shows the crystal rotation magnitude and the components of the rotation axis director between the reference state where the surface is again flat (i.e., initially polished surface), so that in the absence of plastic strain, the integrated displacement $u_{z}$ would be equal to the elevation.

Gradients $G_{x}$ and $G_{y}$ are shown in Figures 14(b) and 14(c) respectively. Crystal rotations at grain boundaries are omitted since they cannot be trusted. Figure 14(d) shows the estimated elevation obtained from integration and after a linear regression to the data has been removed to cancel out the possible global orientation drift and rigid body motions. 


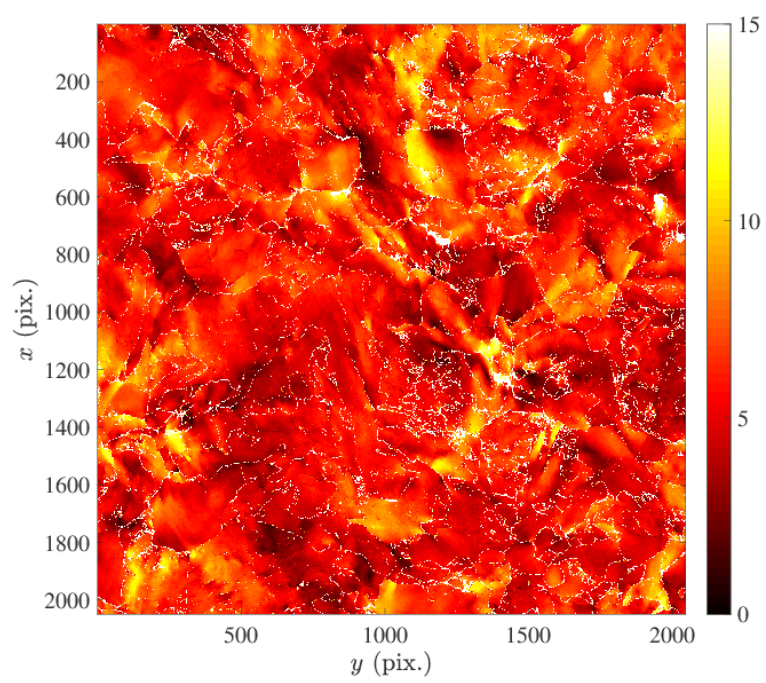

(a) $\omega$

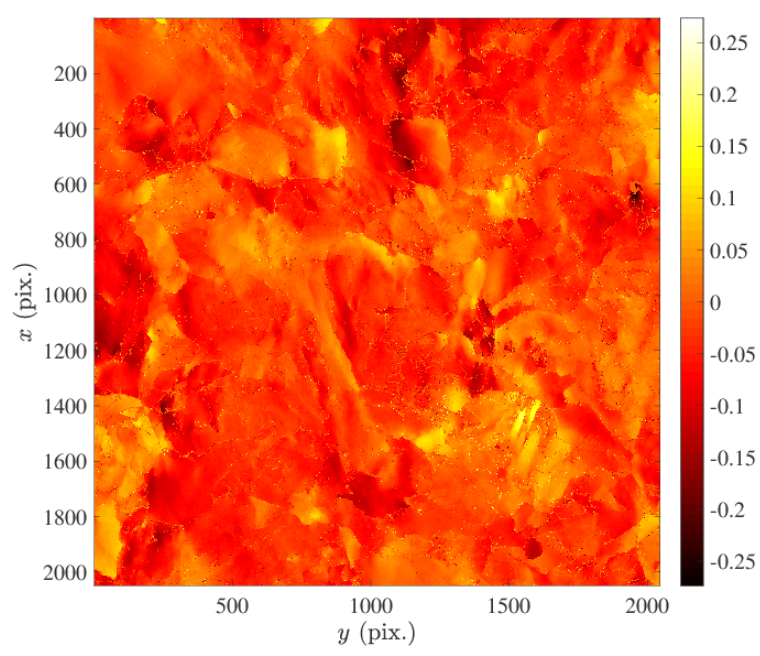

(c)

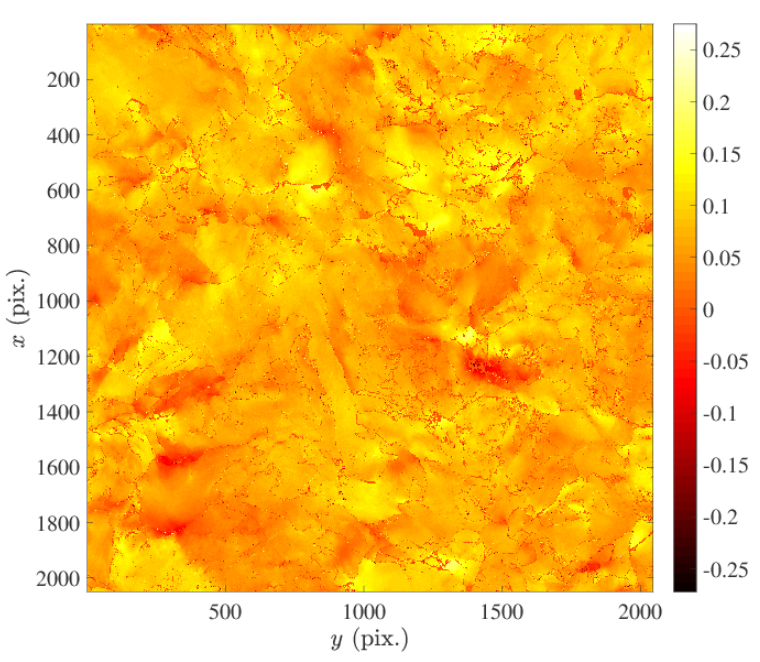

(b)

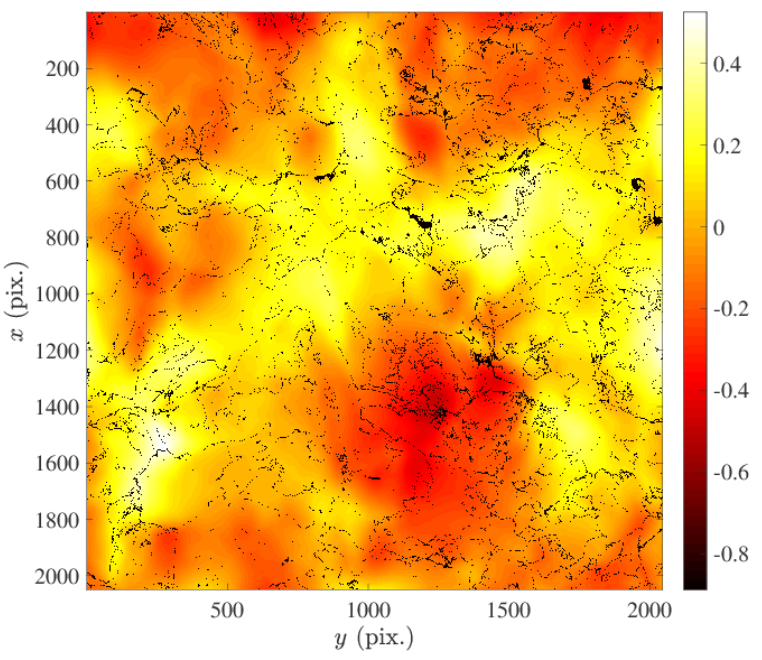

(d)

Figure 14: (a) Rotation magnitude (in degrees) between initial and final states of the tensile test. (b,c) Surface elevation gradient respectively in $x$ and $y$ directions obtained from crystal rotations. (d) Topography (expressed in $\mu m$ ) obtained from integration and subtraction of a linear regression through the data corresponding to orientation indexing "drifts" and rigid body motions. The pixel size is $50 \mathrm{~nm}$ for all images.

\section{Comparison and discussions}

The two methods introduced in the present paper have been tested on the same sample. Thus it is interesting to perform a quantitative comparison of the topography measurement results. Due to time limitation and experimental cost, only $1 / 9$ of the ROI surface was estimated by FIB-milling, while the entire ROI topography was measured by the stereoscopic method (as well as crystal rotation integration). The topography fields of the milled area measured by tilt-imaging and FIB (with FFT smoothing) are shown in Figures 15(a) and 15(b) respectively. Figure $15(\mathrm{c})$ shows the difference between tilting-image and FFT filtered 3D-EBSD measurements. It is observed that the first two methods give very consistent answers. The remaining differences are believed to be due to the lost peak topography information caused by insufficient Pt layer thickness. Note that since the reference plane may not be identical in the two measurements, a regression with a plane has been subtracted from the difference (this is equivalent to changing the reference plane $z=0$ in one measurement in order to match the other one at best). Consequently, the tilt-imaging method is deemed validated, in spite of the very oblique incidence angle. 


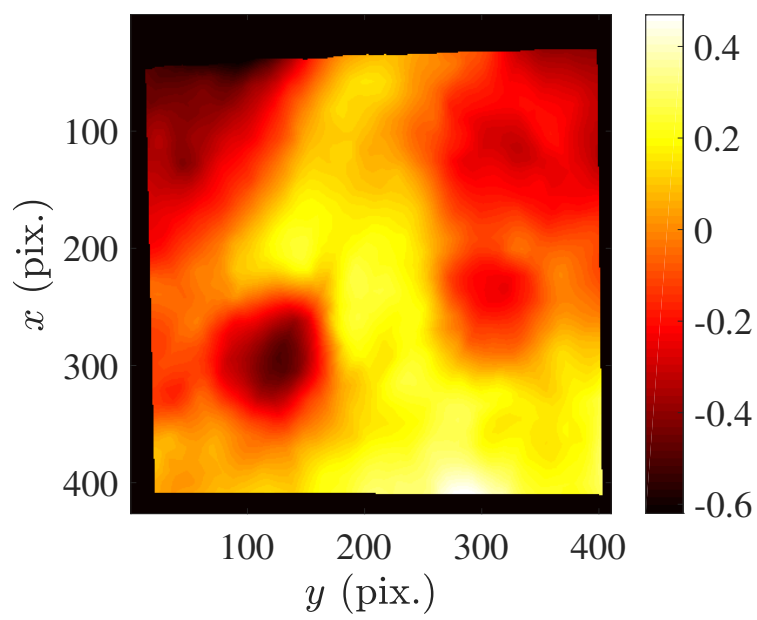

(a)

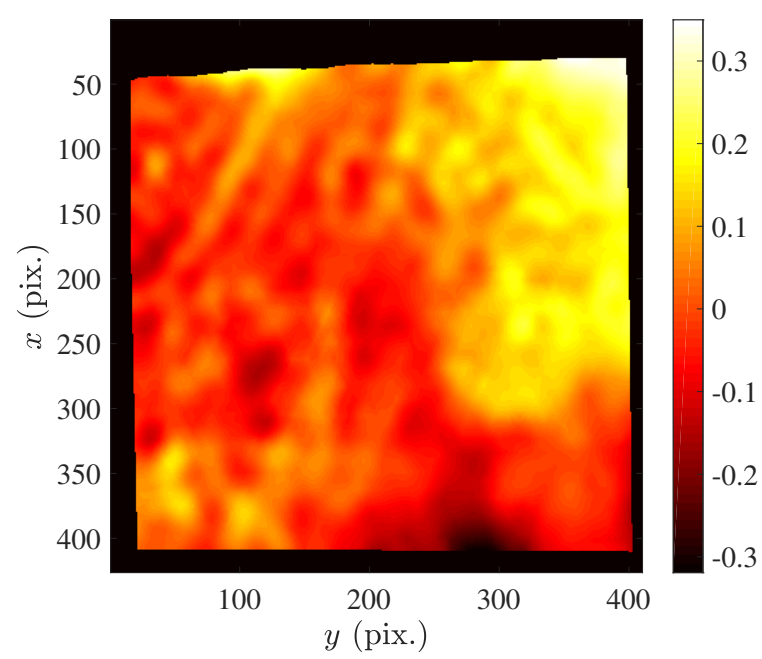

(c)

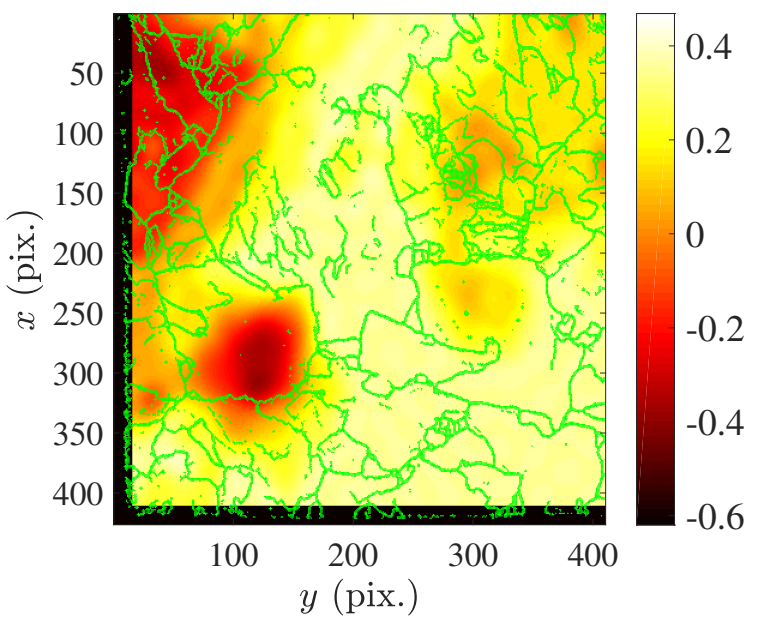

(b)

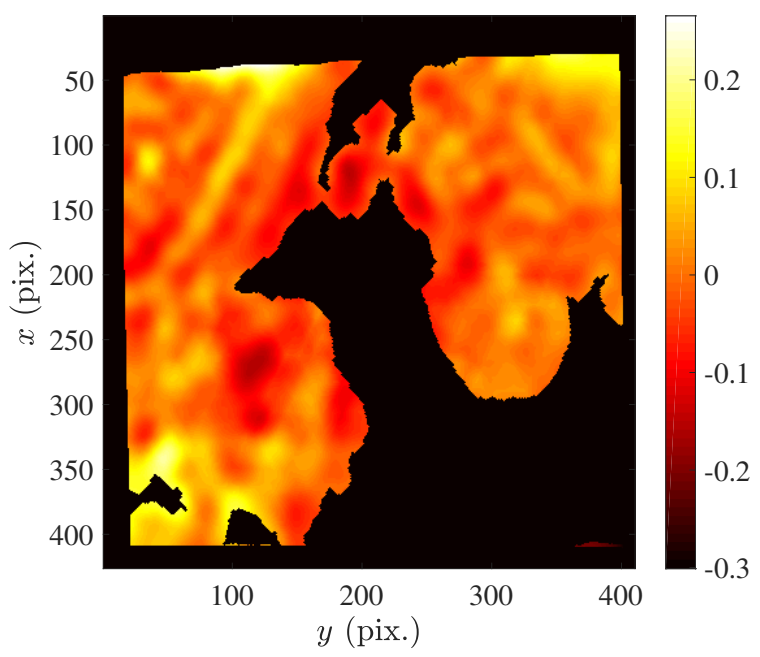

(d)

Figure 15: Topography field of the milled area measured by tilt-imaging (a) and FIB with FFT smoothing (b). (c) Difference between FFT smoothed FIB and tilt-imaging topography measurements. (d) Reliable part of (c) after the subtraction of a plane. The unit of topography is $\mu \mathrm{m}$ and the pixel size is $100 \mathrm{~nm}$ for all images.

Over the reliable part of the measurement (i.e., below the initially emerged topography shown in Figure $15(\mathrm{~d})$, the RMS difference in topography is measured to be $46 \mathrm{~nm}$. The uncertainty of the tilt-imaging method being estimated to be $8.1 \mathrm{~nm}$, the topography measurement uncertainty is about $45 \mathrm{~nm}$ for the FIBEBSD method, as opposed to an RMS of topography roughness of $188 \mathrm{~nm}$. Knowing that the pixel size in SEM imaging is $42 \mathrm{~nm}$, and the voxel size in FIB-EBSD $100 \mathrm{~nm}$, the topography measurement uncertainty is less than the SEM imaging resolutions. This excellent agreement opens up the field of topography measurement to all SEMs equipped with an EBSD setup. Although the principles are far from new, the recourse to such high tilt angles is unusual, and global DIC used herein together with a well-suited speckle patterning provide very robust and accurate topography measurements.

The topographies measured by tilt-imaging and rotation-integration methods, after correction of coordinate distortions, are shown in Figures $16(\mathrm{a})$ and $16(\mathrm{~b})$ respectively. Similar results are observed since the two topography fields have a Pearson correlation coefficient of 0.64 . This is a proof that, even after filtering out with low order polynomials, the rotation-integration method captures part of the topography. However, the RMS roughness of the topography measured by rotation-integration is equal to $171 \mathrm{~nm}$, while that for tilt-imaging topography is equal to $188 \mathrm{~nm}$. Knowing that the rotation-integration method only measures the topography 
due to elastic deformations, it is concluded that plasticity-induced and elasticity-induced topographies are mostly along the same direction and have comparable orders of magnitude.

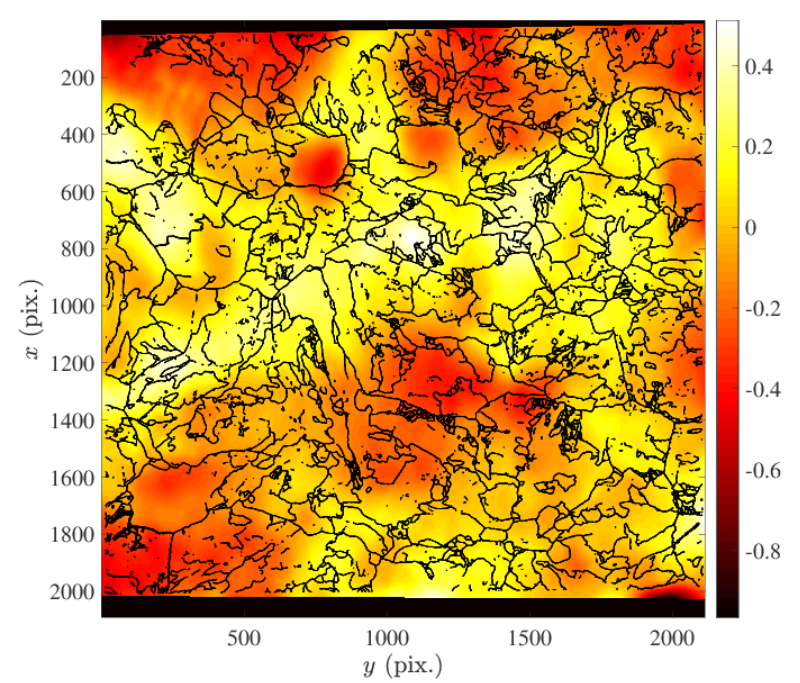

(a)

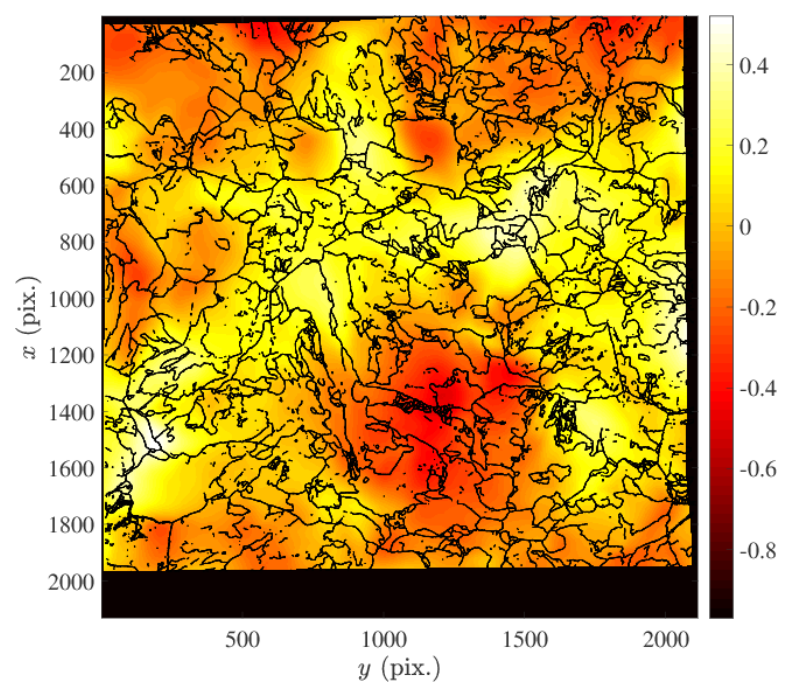

(b)

Figure 16: Comparison of topographies (expressed in $\mu \mathrm{m}$ ) calculated by (a) tilt-imaging and (b) rotation-integration methods. The pixel size is $50 \mathrm{~nm}$ for all images.

Last, an evaluation of each presented method is proposed:

- 3D FIB-EBSD is very simple in its principle and data processing is straightforward, thereby resulting in very reliable three-dimensional microstructures including the surface topography. However, the method is by nature destructive and can only be performed postmortem. Besides, the experimental setup is much more involved (i.e., it requires many operations and the duration of the test is much longer than for the other two methods). Additionally, the measurement resolution is the thickness of each FIB layer, and cannot offer the sub-pixel resolution provided by DIC. An interesting prospect of resolution improvement is to extract more topography information from a FIB slice, such as taking images probing different depths after each FIB removal.

- Stereoscopic tilt-imaging is the fastest method. Its accuracy depends on the size and density of the surface speckles as well as the positioning of the sample in horizontal and tilted configurations. The tilt angle is an essential parameter in the measurement, namely, large angles enhance the magnification of the displacement field issued from the topography during tilt, as demonstrated in previous works [17. However, large $\theta$ angles also yield a more severe contraction effect, leading to poorer resolution in the direction perpendicular to the tilt axis. Moreover, large angles may induce shadows that preclude topography reconstruction in these regions. Therefore, a compromise has to be made in the choice of $\theta$. It is recommended that for very rough surfaces $\theta$ should be small, while for flatter and well-marked surfaces $\theta$ can be set between $40-70^{\circ}$. It should be noted that this method can also be used in other imaging equipments, such as optical cameras and microscopes. A key feature is here the possibility to reveal the surface patterning (i.e., speckles) that circumvent gray level variation issues.

- Rotation-integration can only be applied for samples that have EBSD acquisitions before and after the process by which roughness has appeared (e.g., mechanical test). In theory, the procedure can only give access to the roughness that is due to crystal rotations (and not plastic flow). An integration algorithm combining gradients in $x$ and $y$ directions helps to minimize the effect of (high frequency) Gaussian noise of 
the crystallographic orientations. However, a low frequency orientation error has been observed. A highpass filter (i.e., subtraction of low order polynomial fit) may cancel out or dampen this artifact. However, this is at the risk of removing a significant part of the signal so that the method should be considered with care. Nonetheless, it provides an interesting piece of information distinct from the previous methods, which allows roughness induced by plastic flow and by crystal rotation to be distinguished. This method may benefit from more stable EBSD acquisitions (i.e., less orientation "drift"), or with more precise orientation data, as for example results obtained from high resolution EBSD [38].

\section{Conclusion}

Two very different methods for measuring topographies with SEM images were proposed herein exploiting different modalities. They were tested on a strained sample subjected to a tensile test, conducted in situ in an SEM chamber. The surface topography features were of the order of $1 \mu \mathrm{m}$.

FIB-SEM and tilt-imaging methods measured the overall topography, and provided very consistent results. Their standard uncertainties were evaluated to be of the order of $30 \mathrm{~nm}$. The rotation-integration method was designed to evaluate the topography changes without inelastic strains between two EBSD acquisitions. The resulting topography that differed from the previous two methods showed the effect of plastic flow in the generation of surface roughness in the mechanical test.

These approaches broaden the wealth of information that can be extracted from the different modalities offered by scanning electron microscopy. Last, one of them (based upon stereoscopic tilt-imaging) can be performed at no additional cost with standard SEMs.

\section{Acknowledgments}

The authors acknowledge the financial support of Euratom research and training program 2014-2018 SOTERIA under grant agreement No. 661913. This paper reflects only the authors' view and that the Commission is not responsible for any use that may be made of the information it contains. They also thank Dr. Jan Neggers for constructive advices. 
[1] G. Mendonça, D. Mendonça, F. Aragão, L. Cooper, Advancing dental implant surface technology - from micron- to nanotopography, Biomaterials 29 (28) (2008) 3822-3835. doi:https://doi.org/10.1016/j . biomaterials.2008.05.012.

[2] D. Novovic, R. Dewes, D. Aspinwall, W. Voice, P. Bowen, The effect of machined topography and integrity on fatigue life, International Journal of Machine Tools and Manufacture 44 (2) (2004) 125 - 134. doi: https://doi.org/10.1016/j.ijmachtools.2003.10.018.

[3] S. Morita, M. Abe, K. Yokoyama, Y. Sugawara, Defects and their charge imaging on semiconductor surfaces by noncontact atomic force microscopy and spectroscopy, Journal of Crystal Growth 210 (1) (2000) 408 415. doi:https://doi.org/10.1016/S0022-0248(99)00720-4.

[4] C. Kahloun, R. Badji, B. Bacroix, M. Bouabdallah, Contribution to crystallographic slip assessment by means of topographic measurements achieved with atomic force microscopy, Materials Characterization 61 (9) (2010) 835 - 844. doi:https://doi.org/10.1016/j.matchar.2010.04.011.

[5] P. Franciosi, L. Le, G. Monnet, C. Kahloun, M.-H. Chavanne, Investigation of slip system activity in iron at room temperature by SEM and AFM in-situ tensile and compression tests of iron single crystals, International Journal of Plasticity 65 (2015) 226 - 249. doi:http://dx.doi.org/10.1016/j.ijplas. 2014.09 .008 .

[6] K. J. Ramos, D. E. Hooks, D. F. Bahr, Direct observation of plasticity and quantitative hardness measurements in single crystal cyclotrimethylene trinitramine by nanoindentation, Philosophical Magazine 89 (27) (2009) 2381-2402. arXiv:http://dx.doi.org/10.1080/14786430903120335, doi:10.1080/ 14786430903120335 .

[7] Q. Shi, F. Latourte, F. Hild, S. Roux, Backtracking depth-resolved microstructures for crystal plasticity identification - Part 1: Backtracking microstructures, JOM 69 (12) (2017) 2810-2818. doi:10.1007/ s11837-017-2585-2.

[8] Q. Shi, F. Latourte, F. Hild, S. Roux, Backtracking depth-resolved microstructures for crystal plasticity identification - Part 2: Identification, JOM 69 (12) (2017) 2803-2809. doi:10.1007/s11837-017-2586-1.

[9] A. P. Tafti, A. B. Kirkpatrick, Z. Alavi, H. A. Owen, Z. Yu, Recent advances in 3D SEM surface reconstruction, Micron 78 (Supplement C) (2015) 54 - 66. doi:https://doi.org/10.1016/j.micron.2015.07.005.

[10] C. A. Volkert, A. M. Minor, Focused ion beam microscopy and micromachining, MRS bulletin 32 (5) (2007) $389-399$.

[11] S. Reyntjens, R. Puers, Focused ion beam induced deposition: fabrication of three-dimensional microstructures and Young's modulus of the deposited material, Journal of Micromechanics and Microengineering $10(2)(2000) 181$.

[12] M. Groeber, B. Haley, M. Uchic, D. Dimiduk, S. Ghosh, 3D reconstruction and characterization of polycrystalline microstructures using a FIB-SEM system, Materials Characterization 57 (4-5) (2006) 259 - 273. doi:http://dx.doi.org/10.1016/j.matchar.2006.01.019.

[13] N. Zaafarani, D. Raabe, R. Singh, F. Roters, S. Zaefferer, Three-dimensional investigation of the texture and microstructure below a nanoindent in a Cu single crystal using 3D EBSD and crystal plasticity finite element simulations, Acta Materialia 54 (7) (2006) 1863-1876. doi:https://doi.org/10.1016/j .actamat. 2005. 12.014 
[14] M. Calcagnotto, D. Ponge, E. Demir, D. Raabe, Orientation gradients and geometrically necessary dislocations in ultrafine grained dual-phase steels studied by 2D and 3D EBSD, Materials Science and Engineering: A 527 (10-11) (2010) 2738 - 2746. doi:http://dx.doi.org/10.1016/j.msea.2010.01.004.

[15] J. Guyon, N. Gey, D. Goran, S. Chalal, F. Pérez-Willard, Advancing FIB assisted 3D EBSD using a static sample setup Ultramicroscopy 161 (2016) 161 - 167. doi:http://dx.doi.org/10.1016/j.ultramic. 2015.11.011.

[16] G. Koenig, W. Nickel, J. Storl, D. Meyer, J. Stange, Digital stereophotogrammetry for processing SEM data, Scanning 9 (5) (1987) 185-193. doi:10.1002/sca.4950090502.

[17] W. Lockwood, A. Reynolds, Use and verification of digital image correlation for automated 3D surface characterization in the scanning electron microscope, Materials Characterization 42 (2) (1999) 123 - 134. doi:https://doi.org/10.1016/S1044-5803(98)00052-7.

[18] T. Zhu, M. A. Sutton, N. Li, J.-J. Orteu, N. Cornille, X. Li, A. P. Reynolds, Quantitative stereovision in a scanning electron microscope, Experimental Mechanics 51 (2011) 97-109.

[19] A. Geiger, M. Roser, R. Urtasun, Efficient large-scale stereo matching, in: Asian conference on computer vision, Springer, 2010, pp. 25-38.

[20] S. Yan, A. Adegbule, T. C. Kibbey, A hybrid 3D SEM reconstruction method optimized for complex geologic material surfaces, Micron 99 (Supplement C) (2017) 26 - 31. doi:https://doi.org/10.1016/j . micron.2017.03.018.

[21] E. Binaghi, I. Gallo, G. Marino, M. Raspanti, Neural adaptive stereo matching, Pattern Recognition Letters 25 (15) (2004) 1743 - 1758. doi:https://doi.org/10.1016/j.patrec.2004.07.001.

[22] A. P. Tafti, J. D. Holz, A. Baghaie, H. A. Owen, M. M. He, Z. Yu, 3DSEM++: Adaptive and intelligent 3D SEM surface reconstruction, Micron 87 (Supplement C) (2016) 33 - 45. doi:https://doi.org/10. $1016 /$ j.micron.2016.05.004.

[23] J. Lebiedzik, Multiple detecter method for quantitative determination of microtopography in the SEM, Scanning Electron Microscopy (1975) 181-188.

[24] J. Lebiedzik, An automatic topographical surface reconstruction in the SEM, Scanning 2 (4) (1979) 230237. doi:10.1002/sca.4950020405.

[25] B. K. Horn, Understanding image intensities, Artificial intelligence 8 (2) (1977) 201-231.

[26] T. Suganuma, Measurement of surface topography using SEM with two secondary electron detectors, Journal of Electron Microscopy 34 (4) (1985) 328-337. doi:10.1093/oxfordjournals.jmicro.a050525.

[27] Q. Shi, F. Latourte, F. Hild, S. Roux, Quaternion correlation for tracking crystal motions, Measurement Science and Technology 27 (9) (2016) 095006. doi:https://doi.org/10.1088/0957-0233/27/9/095006.

[28] P. Howell, Taking, presenting and treating stereo data from the SEM, Scanning electron microscopy 1975 (1975) 697-706.

[29] A. Boyde, Quantitative photogrammetric analysis and qualitative stereoscopic analysis of SEM images, Journal of Microscopy 98 (3) (1973) 452-471. doi:10.1111/j.1365-2818.1973.tb03846.x. 
[30] H. Teyssedre, S. Roux, G. Régnier, A. Tracz, Filtering out slow-scan drifts in atomic force microscopy images, The Journal of Strain Analysis for Engineering Design 46 (5) (2011) 361-367. arXiv:http://dx. doi.org/10.1177/0309324711401794, doi:10.1177/0309324711401794.

[31] Q. Shi, S. Roux, F. Latourte, F. Hild, D. Loisnard, N. Brynaert, On the use of SEM correlative tools for in situ mechanical tests, Ultramicroscopy 184 (Part A) (2018) 71 - 87. doi:https://doi.org/10.1016/ j.ultramic.2017.08.005.

[32] Z. Tomičević, F. Hild, S. Roux, Mechanics-aided digital image correlation, Journal of Strain Analysis for Engineering Design 48 (2013) 330-343. doi:10.1177/0309324713482457.

[33] G. Nolze, Image distortions in SEM and their influences on EBSD measurements, Ultramicroscopy 107 (2-3) (2007) 172 - 183. doi:http://dx.doi.org/10.1016/j.ultramic.2006.07.003.

[34] P. Munroe, The application of focused ion beam microscopy in the material sciences, Materials Characterization 60 (1) (2009) 2 - 13. doi:https://doi.org/10.1016/j.matchar.2008.11.014.

[35] M. A. Groeber, M. A. Jackson, DREAM.3D: A digital representation environment for the analysis of microstructure in 3D, Integrating Materials and Manufacturing Innovation 3 (1) (2014) 5. doi:10.1186/ 2193-9772-3-5.

[36] R. T. Frankot, R. Chellappa, A method for enforcing integrability in shape from shading algorithms, IEEE Transactions on Pattern Analysis and Machine Intelligence 10 (4) (1988) 439-451. doi:10.1109/34.3909.

[37] J. Alkorta, Limits of simulation based high resolution EBSD, Ultramicroscopy 131 (2013) 33 - 38. doi: http://dx.doi.org/10.1016/j.ultramic.2013.03.020

[38] A. Wilkinson, G. Meaden, D. Dingley, High-resolution elastic strain measurement from electron backscatter diffraction patterns: New levels of sensitivity, Ultramicroscopy 106 (4-5) (2006) 307 - 313. doi:http: //dx.doi.org/10.1016/j.ultramic.2005.10.001. 